Article

\title{
The Role of Gender Diversity in Downside Risk: Empirical Evidence from Vietnamese Listed Firms
}

\author{
Cong-Duc Tran ${ }^{1} \mathbb{}$, Minh-Tuan Phung ${ }^{1, *}$, Fu-Ju Yang ${ }^{2}$ and Yi-Hsien Wang ${ }^{2}$ \\ 1 Faculty of Business Administration, Ton Duc Thang University, 19 Nguyen Huu Tho st., Tan Phong Ward, \\ District 7, Ho Chi Minh City 729000, Vietnam; trancongduc@tdtu.edu.vn \\ 2 Department of Banking \& Finance, Chinese Culture University, 55, Hwa-Kang Rd., Yang-Ming-Shan, \\ Taipei 11114, Taiwan; fuju@faculty.pccu.edu.tw (F.-J.Y.); holland@mail2000.com.tw (Y.-H.W.) \\ * Correspondence: phungminhtuan@tdtu.edu.vn
}

Received: 20 April 2020; Accepted: 1 June 2020; Published: 8 June 2020

\begin{abstract}
Corporate governance has been marked as an important component of the fast-growing pace of the Vietnam economy recently. Aligning with the concern that gender plays a critical role in corporate management, this study aims to reveal the entire benefits of the appearance of women on board to reduce downside risk in the frontier countries. By using a unique dataset from Vietnamese listed firms and appropriate econometric methods, we present tight results that the presentation of women at firm management level is more likely to lessen the downside risk. In particular, female non-senior reduces all risk attributes, whereas the presence of women in executive board decrease firm risk only. Furthermore, the decreasing of individual and systemic risk are additionally driven by female Chief Executive Officer (CEO) and chairman. We found strong evidence that female leadership may decrease risk-taking in the low-risk firms at all managerial levels but not in the case of a female CEO. In summary, by examining data from specific frontier areas such as Vietnam, we confirm that the role of female leadership in terms of reducing the downside risk depend on their power managerial levels and the firm-risk behavior.
\end{abstract}

Keywords: corporate governance; gender diversity; downside risk; parametric method

JEL Classification: G30; G32; M16

\section{Introduction}

The twenty-first century has been marked as the most glorious period for the massive growth of Asian economics that implicates an increase in pressure on corporate governance for firm managers. Among them, gender equality in the boardroom has been raised as one of the concerns that impact corporate risk-taking [1]. Gender equality in the board is noticed as the main factor in corporate governance that improves firm performance [2]. Even though the proposition of women in management globally increased by 5\% to $29 \%$ in 2019 , it does not represent the scenario in Asian, where the role of women in business has still been underestimated (following the annual report of Grant Thornton, An Instinct for Growth, in 2019) [3].

Besides, as the weakness of internal corporate governance leads to the increase in agency cost, it is mentioned as the root of the recent financial crisis [4,5]. Indeed, the firm with good corporate governance is expected to perform better due to the lower cost capital and default risk [6]. In line with that viewpoint, the downside risk or excessive risk-taking behavior is highlighted as the main contribution to the cause of crisis due to the risk bearing of managers [7,8]. With the importance of corporate governance in protecting shareholders from undesirable downturn of stock market, the board 
of directors is, therefore, as one of the essential functions that control governance mechanism and risk-taking behavior.

Furthermore, there is a significant amount of research analyzing the role of good corporate governance to enhance firm performance $[9,10]$. However, the regarded papers investigating the relationship between gender diversity in board and risk management, specifically downside risk, are still in debate [11-13]. In particular, it is necessary to clarify the connection between the risk attributes of downside risk and the power of women in all firm managerial levels instead of assuming such risks by only measuring the proportion of women on board [1]. Corporate risk-taking is a primary function to achieve the economic reward, and maximize not only shareholder benefits, but also board responsibilities in determining the wiliness to take a risk in pursuing strategic objectives. Therefore, the risk is described as an important indicator defining a firm's competitiveness in a specific industry [14]. According to the behavioral agency problems [15], our measures of risk portrait both individual risk and systemic risk $[5,16]$. In details, individual risk represents the value increasing strategies that the board of directors follows towards increasing shareholder's wealth, whereas systematic risk refers to the risk inherent to the entire market or market segment that causes the collapse of many individual firms.

Intensively, our study addresses the critical question of how gender diversity in firm management influences downside risk by looking into the context of a frontier market. Thus, it extends the literature by several important contributions. First, this research is one of the first to relate corporate governance to downside risk. Since the Basel Committee (Basel Committee on Banking Supervision) required measuring downside risk by value at risk (VaR) in 1996, VaR has become common in use and been applied beyond the financial sector $[17,18]$. In addition, using VaR is appealing because of its ability in describing the downside risk with a single number $[19,20]$. Furthermore, although VaR is merit to reflex the potential loss and its capability in practice, its measurement is still complex [1]. There are a vast of methods to measure VaR such as parametric [21]; non-parametric [22]; and semi-parametric [23]. In consideration of these measurements, this research provides the empirical results on applying to generalize hyperbolic distribution (GHD)-based parametric method to compute VaR and expected shortfall (ES), which is mentioned to be coherent risk measurement [24,25].

Second, whereas the value of corporate governance has been emphasized in the firm performance relationship, the empirical evidence on the relationship between gender diversity and downside risk is considered as complicated [26]. Researchers are conceivably inclined to focus on internal governance [27-29], ownership concentration [30,31], and even comparing different aspects of governance mechanisms [6] affecting corporate risk. Different from previous studies, this paper focuses on clarifying the role of women in top management and downside risk in frontier Asian markets such as Vietnam. Although the gender diversity on board of director leads to reduce corporate risk [9,11], women are normally appointed to the non-executive position [32], which alleviates their involvement role in the strategic decision-making process even inboard [33,34]. Therefore, we argue that firms with a higher proportion of female managers assume lower excessive risk-taking behavior, but it depends on their power managerial levels.

Third, although the concept of corporate governance has been widely analyzed, there is a lack of research focusing on frontier markets such as Vietnam [35,36]. In particular, the framework of corporate governance in Vietnam has been in an early stage of development, even its economy was reformed in 1991 [36]. In line with that view, Vietnam is mentioned as an under-developed, weak national governance system [37], and Vietnamese firms are characterized by having highly concentrated ownership with a low proportion of female managers $[31,38]$. In such conditions, especially with poor corporate governance environment, gender diversity can serve as a corporate governance mechanism that can potentially affect firm risk-taking [11]. In addition, Vietnam has still been marked as a frontier market from Morgan Stanley Capital International (MSCI) index (According to the annual market classification review of Powering better investment decisions from MSCI in 2019). Serious imperfections like information asymmetries make Vietnamese market as an excellent candidate for 
verifying the impact of firm management on corporate risk-taking. Thus, these results have potential implications for other frontier markets that are poorly governed.

Overall, from the final results, we expect to find the profound significance of female managers on reducing downside risk. Herein, we distinguish the appearance of women in all aspects of managerial positions including senior and non-senior managers; chief executive officer, and chairman. In addition to deal with the endogeneity concern, we then divide women on board of directors into executive and non-executive levels. Thus, we proceed to investigate whether the downside risk is driven by their power that goes with the managerial levels or not [33]. Finally, we test whether it is different from gender diversity in determining risk-taking in high and low firm risks.

Eventually, the structure for the rest of this paper is organized into the following parts: the current literature of gender diversity in the boardroom is discussed in Section 2; data and methodology are shown in Section 3; empirical results and its implication are presented in Sections 4 and 5 concludes this study.

\section{Literature Review}

In the framework of the agency problem, the association of executive managers and risk-averse is normally explained by their reputation and employment $[39,40]$. However, the managers may ignore the principles of management function to take more risk due to the greater managerial incentives [41,42]. Thus, refs. [8,43] show that the financial crisis in 2008 is mainly caused by excessive risk-taking behavior from executive managers due to greater performance-based managerial incentives. Therefore, the tightly internal mechanisms play an essential role in monitoring and enhancing the quality of corporate governance as well as reducing the interest difference of stakeholders [40]. Moreover, ref. [31] recently mention the role of corporate governance in corporate firm's risk-taking in frontier equity markets characterized by high ownership concentration and poor governance mechanisms.

Although the tighter regulations help the governance mechanism effectively reduce the agency problems, they may cause negative effects such as moral hazard in the risk investment system. While shareholders are limited in assets, tighter regulations reduce their involvement in firm strategy decisions $[44,45]$. Following this, intensive government regulations may weaken external mechanisms [46]. Ref. [47] acknowledged that the cost of the hostile takeover was the important reason for the avoidance of firms, thus the internal mechanisms (e.g., the board of directors, managerial characteristics) become adapted factors in reducing agency problems. Ref. [48] explored this idea and found the negative impact of board size and power concentration of Chief Executive Officer (CEO) play on agency problems. Ref. [49] followed up on [48] research suggesting that the difference of board structure (e.g., more appearance of non-executive directors and more powerful CEO) reduces the agency cost and enhances the efficiency incorporate risk-taking. Recently, refs. [2,50] emphasized that the role of gender diversity in boardroom raises the confidence of investors due to the expectation that women would enhance internal mechanisms. In summary, by focusing on the role of women to improve firm internal mechanisms, the effect of gender diversity in the boardroom on corporate risk-taking is particularly discussed in the next following section.

\subsection{Gender Diversity and Corporate Risk-Taking}

The current literature frequently shows that the distinct gender stereotypes of men and women which are pointed as the main reasons to diversify risky behavior between them. Both risk aversion and risk-bearing are considered. Ref. [51] found that men tend to invest twice as less of their wealth in stocks and prepare their retirement plans in a less conservative way than women. Therefore, women are assumed to involve less in risk-taking behaviors than male counterparts [52]. In the context of a group, ref. [53] showed that women are less risky than a man in their willingness to lead the team decision. Particularly, ref. [54] detected that women are more conservative in risk attributes as well as ambiguity than their counterparts in the financial areas which attitude towards the risk of professional investors. 
Furthermore, the difference between compensation and incentives may be employed to the documented association between gender and risk-taking. Specifically, low-risk firms may be more likely to offer fixed pay contracts and more likely to attract female executives. Consistent with this argument, ref. [55] used the sample of Italian firms to reveal that more risk-averse and less talented managers tend to match with firms offering low-powered incentives. As [56] argued, with respect to firm performance and firm risk, the influence of female managers may indirectly result in firms having fewer returns (e.g., risk-averse) and fewer huge losses (having more stable performance). Such a risk-return paradox may be the result of differences in management capabilities brought on through the different gender characteristics. Ref. [1] contributed to that viewpoint as they found out that the appearance of women in the boardroom has a negative impact on risk in financial institutions in the European area. In contrast, ref. [12] showed that risk-taking behavior is increased by not only by younger executive teams but also the higher proportion of female executives in the German financial sector. Ref. [26] showed that the proportion of women onboard leads to an increase in risk-taking. In addition, the low proportion of women on board can be explained by their conservativeness and sensitivity that could be inefficient managers [57].

Despite the fact that the interest in gender difference in governance attributes has been amplified in recent years, these have not so many papers explored carefully the relation between women on executive positions and corporate risk-taking. Therefore, in line with the view that higher proportion of women in firm management increase firm financial performance and lower downside risk [1,58], we then expect to find the negative impact between gender diversity on corporate risk-taking in Vietnam. We propose our first hypothesizes following as:

Hypothesis 1a (H1a). The proposition of women on firm management has a negative significant relationship with individual risk.

Hypothesis $\mathbf{1 b} \mathbf{( H 1 b )}$. The proposition of women on firm management has a negative significant relationship with system risk.

Hypothesis 1c (H1c). The proposition of women on firm management has a negative significant relationship with system risk accumulation.

\subsection{Women in Top Executive and Corporate Risk-Taking}

There is a vast evidence which shows that female leadership can improve both internal mechanisms and the firm's external performance [59-61]. Therefore, the attendance of women in board of directors could help the firm reduce coordination and communication problems in the boardroom. By employing the dataset of 638 firms listed from American Fortune 1000, ref. [60] showed the significant connection between the proportion of women on board and firm performance illustrated by Tobin's $Q$ ratio. Ref. [62] found that a higher percentage of women on board increases firm performance which presents by both accountant and market-based ratios. Supporting the perspective that women should be encouraged to participate in a leadership function, ref. [59] argued that female leadership increases firm performance at both accountant and market-based aspects. Furthermore, ref. [63] showed interesting evidence that the proportion of women on board could make a negative effect on firm performance if it reaches the particular number of 30 percent.

We also emphasize that even though the growth of relevant literature in board structure has increased quickly, it is not in the case of gender and corporate risk-taking. In the context of gender equality encouragement in the boardroom, it shows that women leaders are more disciplined, particularistic, and democratic than men in executive function [64]. Aligning with that view, ref. [65] indicated that the unique characteristics of women such as a slight edge over men support firm strategy. Developing from these findings, ref. [61] argued that women should be on board due to their flexibility in internal communication and being less hierarchical. Later, refs. [66,67] agreed that women have 
enough capabilities to make the difference in executive positions with their unique characteristics. Hence, female leadership has the ability to fulfill the board's strategic role.

Regarding market assessment to the female executives, ref. [68] showed the underrated evaluation of market to female CEOs. Furthermore, they also found consistent results that firms with female leadership decrease idiosyncratic risk. The uniqueness of women's characteristics in leadership positions is also clearly clarified in the study of [69] that companies with female leaders us less leverage, less volatile earnings, and higher opportunity for survival.

Although female employees have been encouraged to participate more in executives, the empirical evidence on the relationship between female executive managers and corporate risk-taking has not made such distinction. We, therefore, expect the negative relationship between female manager and all risk attributes of downside risk in Vietnam and propose our second hypothesis as follow:

Hypothesis 2a (H2a). The representation of women as top executive manager has a negative significant relationship with individual risk.

Hypothesis $\mathbf{2 b}(\mathbf{H} \mathbf{2} \mathbf{b})$. The representation of women as top executive manager has a negative significant relationship with system risk.

Hypothesis 2c (H2c). The representation of women as top executive manager has a negative significant relationship with system risk accumulation.

\section{Methodology}

\subsection{Data}

Osiris is a confident database for this study because it comprises the massive data of corporate governance from most of the firms listed in the stock market, including the firm's statement for data validity. Therefore, we use Osiris to collect governance mechanism variables, whereas Datastream is a confidential source for the equity data of listed companies. Although Osiris is well-known as a strong database on corporate governance structure, not all values are summarized. For example, we need to handle the missing values manually by including several important variables such as the gender of managers. Specifically, we counted the number of female directors who are still attending on board, and then they are cross-checked through companies' official websites.

This study uses 178 Vietnamese listed companies including 1424 observations that represent seven essential product and manufacturing sectors (especially excepting finance and real estate). The Vietnamese government's massive privatization scheme since 1986 has changed significantly ownership structure in Vietnamese enterprises. Therefore, the listed equity market of Vietnam, which has been available since 2000, constitutes an interesting venue for governance research [31].

Besides, the research period runs from 2010 to 2017, which is relevant to two remarkable events: (1) the period is marked as Vietnamese economic recovery since the happening of the financial crisis in 2009 [70]; and (2) the "law on enterprises" was revised in 2014. The new regulations highlight the important role of internal governance mechanisms in firm performance improvement [31], and the essential role of privatizing state-owned enterprises [71].

\subsection{Variables and Econometric Models}

Since Morgan proposed their RiskMetrics in 1996 (see RiskMetrics Group in 1994) (as stressed in [72], this sort of risk method was proposed in early of 1963 by Baumol and was referred to as the expected gain-confidence limit criterion [73]), value at risk (VaR) (See an internal model-based approach to market risk capital requirements (Bank for International Settlements in 1995)) has widely 
recognized as a compelling tool in monitoring financial risk that goes beyond the financial sector [4]. It is defined as the possibility of maximum loss in a confidence level following as:

$$
V a R_{\alpha}=\inf \{x \in \mathrm{R}: P(X>1) \leq 1=\alpha\}=\inf \left\{x \in \mathrm{R}: F_{X}(x) \geq \alpha\right\}
$$

where $F_{X}$ is the distribution function of the loss.

In this study, we use the generalize hyperbolic distribution (GHD) to calculate VaR and expected shortfall (ES). That distribution is acknowledged to be the comprehensive of downside risk measurement [1,24]. According to [25], GHD can be presented as:

$$
\left.f(x)=a(\lambda, \alpha, \beta, \delta)\left[\delta^{2}+(x-\mu)^{2}\right)^{\frac{\left(\lambda-\frac{1}{2}\right)}{2}}\right] \cdot K_{\lambda-\frac{1}{2}}\left(\alpha \sqrt{\delta^{2}+(x-\mu)^{2}}\right) \exp [\beta(x-\mu)]
$$

where,

$$
a(\lambda, \alpha, \beta, \delta)=\frac{\left(\alpha^{2}-\beta^{2}\right)^{\lambda / 2}}{\sqrt{2 \pi} \alpha^{\lambda-1 / 2} \delta^{\lambda} K_{\lambda}\left(\delta \sqrt{\alpha^{2}-\beta^{2}}\right)}
$$

and $0 \leq|\beta| \leq \alpha ; \mu$ and $\lambda \in \mathrm{R}$, and $\delta>0$. The parameters $\mu$ and $\delta$ representing the location and scale, respectively. The shape parameters, $\alpha$ and $\beta$, correspond to tail heaviness and asymmetry of the density, respectively. $K_{\lambda}$ is a modified Bessel function of the third kind and $x \in \mathrm{R}$.

By considering excessive risk-taking as the main reason contributing to the unsustainability of the market and directly affecting firm's competitiveness, our paper will focus on exploring the role of gender diversity on excessive risk-taking behavior as follow:

$$
\text { Risktaking }_{i, t}=\beta_{0}+\beta_{1} \text { Gender }_{i, t}+\beta_{2} \text { Board }_{i, t}+\beta_{3} \text { Firm }_{i, t}+\varepsilon_{i, t}
$$

where Risktaking is classified by the risk attributes of downside risk. Gender defines gender diversity. Board and Firm represent for board characteristics and firm characteristics respectively. All variable definitions are presented in Table 1.

The model is basically estimated by two-stage least squares (2SLS) with robust standard errors clustered by firm. Potential problems of endogeneity are considered in model estimation. Following [26], we test and confirm that the own variable is truly instrumented by lnage and fbpower (represent for the female senior managers on average of all firms in the same sector) with the null hypothesis of Wooldridge test indicating exogeneity. Further, we apply the Sargan test for over-identifying restrictions to check for instrument exogeneity, where insignificant test statistics indicate that the instruments may be valid. Additionally, we highlight that the proportions of female managers could also be endogenous because of a reverse causality of firm risk and the appointment of the executive managers $[14,74]$. The variables of gender diversity, therefore, have been lagged to alleviate that concern. We further robust our results, even overcome these limitations by applying generalized method of moments (GMM). It is addressed to control for simultaneity concerns, omitted variable bias and the dynamic relationship of current appointed female executive managers and previous realizations of risk [75]. 
Table 1. Variables definition.

\begin{tabular}{|c|c|c|}
\hline Variables & & Definition \\
\hline \multicolumn{3}{|c|}{ Risk measurement } \\
\hline - Individual risk & IR & $\begin{array}{l}\text { Generalized hyperbolic distribution-based value at risk (VaR) at } 99 \% \text { confidence level, derived } \\
\text { from the firm's market price }\end{array}$ \\
\hline - Systemic risk (SR) & SR & $\begin{array}{l}\text { Generalized hyperbolic distribution-based conditional value at risk (CVaR) at } 99 \% \text { confidence } \\
\text { level, derived from the firm's market price }\end{array}$ \\
\hline - Contribution risk (CSR) & CSR & $\begin{array}{l}\text { Calculated by the difference between individual risk (IR) and median possible loss of the system, } \\
\text { conditional on each firm's loss }\end{array}$ \\
\hline \multicolumn{3}{|c|}{ Gender diversity } \\
\hline - Gender of Chairman & Chair & Dummy variable which is equal 1 if the firm's chairman is female and 0 otherwise \\
\hline - Gender of CEO & CEO & Dummy variable which is equal 1 if the firm's CEO is female and 0 otherwise \\
\hline - The proportion of women in senior management & Senior & The ratio of female directors on board of director \\
\hline - The proportion of women in non-senior management & Nonsenior & The ratio of women on the firm's management level \\
\hline - The proportion of women as executive director in the boardroom & Exe & The ratio of female executive directors to board size \\
\hline - The proportion of women as non-executive in the boardroom & Nonexe & The ratio of female non-executive directors to board \\
\hline \multicolumn{3}{|c|}{ Board characteristics } \\
\hline - Senior manager size & Board & Natural logarithms of the number of board of directors \\
\hline - Nonsenior manager size & Manager & Natural logarithms of the number of managers \\
\hline - Chairman age & Achair & Age of chairman to 2017 \\
\hline - CEO age & Aceo & Age of CEO to 2017 \\
\hline • CEO/Chairman duality & Duality & Dummy variable equal to (1) if the position of CEO and Chairman are combined, (0) otherwise. \\
\hline \multicolumn{3}{|c|}{ Firm characteristics } \\
\hline - Size of firm & Size & Size of the firm is equal to the natural logarithm of total assets \\
\hline - Cash flow ratio & Cash & The ratio of cash flow to total assets \\
\hline - Book equity ratio & Bea & The ratio of total book equity to total assets \\
\hline - Tangible assets ratio & Tang & The ratio of tangible assets to total assets \\
\hline - Firm leverage ratio & Lev & The ratio of total debt to total assets \\
\hline - Firm market ratio & MB & The ratio of the market capability to book value \\
\hline - Ownership ratio & Own & The ratio of firm ownership to total equity \\
\hline
\end{tabular}




\subsection{Measurement}

\subsubsection{Dependent Variable}

We conceptualize downside risk that is proxy into three risk attributes including individual risk (IR) and two its sub-components, namely, systemic risk (SR) and systemic contribution risk (CSR). First, we estimated the IR, which is defined as the maximum possible loss of each firm's market value asset at a given confidence level (99\%) (e.g., the GHD-based value at risk/VaR). Second, we calculate SR, which is defined as the maximum possible loss of the system's market assets at a given confidence level (99\%), conditional on each firm's IR (i.e., the GHD-based conditional value at Risk/Expected shortfall). Finally, the CSR of each firm is determined by the difference between IR and SR. The accuracy of the risk calculation is carefully validated by the percentage of violations from [76].

\subsubsection{Key Explanatory Variables}

To explore the impact of female managers on downside risk, we consider the proportion of all women on (1) senior and (2) non-senior management and their position as the firm's representation in law such as (3) chairman and (4) the CEO. In addition to further our findings and test for the validity of regression results, we measure the proportion of executive and non-executive female directors to board size (exe and non-exe, respectively), which estimate whether the impact of female directors on risk-taking is a result of management responsibilities.

\subsubsection{Control Variables}

According to prior literature, we then select a set of control variables and devices into two groups: (1) board characteristic and (2) firm characteristic.

Board characteristics: We used both ages of the chairman (Achair) and CEO (Aceo) to control for the working experience. With regards to the structure of the executive managers, we considered senior and non-senior size (illustrated by Board and Manager respectively), measured by the natural logarithm of the number of women on board and lower-level managers. Furthermore, duality controls for the different from power between chairman and CEO in firm final decision making.

Firm characteristic: It indeed had been found that the existing inconclusive evidence of the female leadership is inadequate for other factors explaining financial performance [77,78]. We thus controlled the size of firms, cash flow, shareholder fund, tangible asset, leverage, growth opportunities, and ownership structure. However, other firm-specific characteristics that are unobservable or unmeasured in our model (e.g., the appointment of a new CEO) could result in an estimation bias [48].

\section{Empirical Results}

\subsection{Data Analysis}

Table 2 shows the descriptive statistics of all the variables. The percentage of observations (firms) with at least one women on the management board is $22 \%$, which are increased to $26.97 \%$ when it refers to non-senior managing level. The proportion of women at the chief executive officer (CEO) position is quite low $(9.55 \%)$, even as chairman of firms $(10.67 \%)$. These numbers are consistent with the report of Thornton (2019). It indicates that the joining of women at the executive level is no more than $30 \%$ compared to their counterparts. The average size of the board of directors (management) is approximately nine directors (six managers). On average, the women hold the executive position lower than their counterparts, even the total number of senior managers is higher than non-senior managers. Moreover, the sample contains the average percentage of major shareholders (own) is $59 \%$. 
Table 2. Sample statistics.

\begin{tabular}{cccccc}
\hline Variable & Obs & Mean & Std. Dev. & Min & Max \\
\hline IR & 1424 & 0.042 & 0.378 & 0.003 & 14.275 \\
SR & 1424 & 0.059 & 0.715 & 0.002 & 26.996 \\
CSR & 1424 & 0.017 & 0.337 & -0.002 & 12.721 \\
Senior & 1424 & 0.220 & 0.167 & 0.000 & 0.750 \\
Nonsenior & 1424 & 0.270 & 0.194 & 0.000 & 0.750 \\
Exe & 1424 & 0.125 & 0.134 & 0.000 & 0.667 \\
Nonexe & 1424 & 0.095 & 0.105 & 0.000 & 0.600 \\
CEO & 1424 & 0.095 & 0.294 & 0.000 & 1.000 \\
Chair & 1424 & 0.107 & 0.309 & 0.000 & 1.000 \\
Manager (Number) & 1424 & 6.416 & 2.880 & 3.000 & 23.000 \\
Board (Number) & 1424 & 8.601 & 3.087 & 4.000 & 25.000 \\
Achair & 1424 & 3.899 & 0.187 & 2.944 & 4.382 \\
Aceo & 1424 & 3.814 & 0.213 & 2.944 & 4.304 \\
Size & 1424 & 20.406 & 1.364 & 16.859 & 24.695 \\
Duality & 1424 & 0.298 & 0.457 & 0.000 & 1.000 \\
Cash & 1424 & 0.101 & 0.252 & 0.000 & 8.466 \\
Bea & 1424 & 0.395 & 0.202 & -0.481 & 0.959 \\
Tang & 1424 & 0.268 & 0.190 & 0.002 & 0.853 \\
Lev & 1424 & 0.274 & 0.197 & 0.000 & 0.869 \\
MB & 1424 & 3.090 & 7.813 & -79.386 & 96.047 \\
Own & 1424 & 0.589 & 0.196 & 0.124 & 0.997 \\
\hline
\end{tabular}

Table 3 presents the correlation matrix between independent variables. All coefficients are generally low, being the highest relevant coefficient between the age of Chairman and CEO (0.437). To check whether a problem of multicollinearity might be present, we perform a variance inflation factor (VIF) test. There are no serious problems of multicollinearity and all the condition indices are below 10 [79]. Furthermore, the mean VIF of all variables is 1.42 , which confirms the validity of variables using in this study.

\subsection{Two-Stage Least Squares (2SLS) Testing}

Table 4 shows the results of 2SLS estimations with robust standard errors clustered by the firm for the three measures of downside risk: individual risk (IR, columns 1-4), systemic risk (SR, columns 5-8), and systemic contribution risk (CSR, columns 9-10). To test H2a and H2b, we distinguish women as chairman is the head of the board of directors while the CEO is the top senior executive. We found that both IR and SR are increased by both female CEO and chairman, but have no significant impact on CSR. Thus, these findings support both $\mathrm{H} 2 \mathrm{a}$ and $\mathrm{H} 2 \mathrm{~b}$, but reject H2c. The significant and negative effect of CEO and chairman to IR and SR regards to the assumption of agency theory with the special characteristics of women on their employment risk consideration [80]. In support of H1a, we provide evidence that the presence of women in firm senior management is negative and significant in determining individual risk, but it does not show a significantly negative relation to SR and CSR. Thus, the results do not support H1b and H1c. According to [2], women are more efficient to improve internal mechanisms. Thus, their appearance of firm management in reducing excessive risk-taking behavior is strongly supported [1].

Among the control variables, the increase in firm size causes a decrease in all risk attributes. However, the positive impact of Bea and Tang shows the logical evidence that firms that have more financial capabilities tend to take more risks than the others. 
Table 3. Correlation matrix.

\begin{tabular}{|c|c|c|c|c|c|c|c|c|c|c|c|c|c|c|c|c|c|c|c|}
\hline & & (1) & (2) & (3) & (4) & (5) & (6) & (7) & (8) & (9) & (10) & (11) & (12) & (13) & (14) & (15) & (16) & (17) & (18) \\
\hline (1) & Senior & 1 & & & & & & & & & & & & & & & & & \\
\hline (2) & Nonsenior & $\begin{array}{l}0.382 \\
(0.000)\end{array}$ & 1 & & & & & & & & & & & & & & & & \\
\hline (3) & Exe & $\begin{array}{l}0.775 \\
(0.000)\end{array}$ & $\begin{array}{l}0.308 \\
(0.000)\end{array}$ & 1 & & & & & & & & & & & & & & & \\
\hline (4) & Nonexe & $\begin{array}{l}0.697 \\
(0.000) \\
\end{array}$ & $\begin{array}{l}0.2124 \\
(0.000)\end{array}$ & $\begin{array}{l}-0.046 \\
(0.085)\end{array}$ & 1 & & & & & & & & & & & & & & \\
\hline (5) & CEO & $\begin{array}{l}0.228 \\
(0.000) \\
\end{array}$ & $\begin{array}{c}0.311 \\
(0.000) \\
\end{array}$ & $\begin{array}{l}0.308 \\
(0.000) \\
\end{array}$ & $\begin{array}{l}-0.045 \\
(0.085) \\
\end{array}$ & 1 & & & & & & & & & & & & & \\
\hline (6) & Chair & $\begin{array}{l}0.343 \\
(0.000) \\
\end{array}$ & $\begin{array}{c}0.119 \\
(0.000) \\
\end{array}$ & $\begin{array}{l}0.212 \\
(0.000) \\
\end{array}$ & $\begin{array}{c}0.181 \\
(0.000) \\
\end{array}$ & $\begin{array}{l}0.321 \\
(0.000) \\
\end{array}$ & 1 & & & & & & & & & & & & \\
\hline (7) & Manager & $\begin{array}{l}-0.100 \\
(0.000)\end{array}$ & $\begin{array}{l}-0.057 \\
(0.032)\end{array}$ & $\begin{array}{l}0.311 \\
(0.000) \\
\end{array}$ & $\begin{array}{l}0.458 \\
(0.000)\end{array}$ & $\begin{array}{l}-0.061 \\
(0.020)\end{array}$ & $\begin{array}{l}-0.056 \\
(0.033)\end{array}$ & 1 & & & & & & & & & & & \\
\hline (8) & Board & $\begin{array}{l}0.099 \\
(0.000)\end{array}$ & $\begin{array}{l}0.008 \\
(0.777)\end{array}$ & $\begin{array}{l}0.119 \\
(0.000) \\
\end{array}$ & $\begin{array}{l}0.029 \\
(0.280)\end{array}$ & $\begin{array}{l}-0.001 \\
(0.963)\end{array}$ & $\begin{array}{l}-0.025 \\
(0.339) \\
\end{array}$ & $\begin{array}{l}0.413 \\
(0.000)\end{array}$ & 1 & & & & & & & & & & \\
\hline (9) & Achair & $\begin{array}{l}-0.199 \\
(0.000)\end{array}$ & $\begin{array}{l}-0.104 \\
(0.000)\end{array}$ & $\begin{array}{l}-0.056 \\
(0.032) \\
\end{array}$ & $\begin{array}{l}0.050 \\
(0.058)\end{array}$ & $\begin{array}{l}-0.045 \\
(0.089)\end{array}$ & $\begin{array}{l}-0.071 \\
(0.007) \\
\end{array}$ & $\begin{array}{l}-0.067 \\
(0.011)\end{array}$ & $\begin{array}{l}-0.064 \\
(0.015)\end{array}$ & 1 & & & & & & & & & \\
\hline (10) & Aceo & $\begin{array}{l}-0.094 \\
(0.000)\end{array}$ & $\begin{array}{l}0.002 \\
(0.951)\end{array}$ & $\begin{array}{l}0.008 \\
(0.777) \\
\end{array}$ & $\begin{array}{l}0.055 \\
(0.037)\end{array}$ & $\begin{array}{l}-0.045 \\
(0.090)\end{array}$ & $\begin{array}{l}-0.044 \\
(0.094) \\
\end{array}$ & $\begin{array}{l}-0.018 \\
(0.506)\end{array}$ & $\begin{array}{l}-0.027 \\
(0.300)\end{array}$ & $\begin{array}{l}0.437 \\
(0.000)\end{array}$ & 1 & & & & & & & & \\
\hline (11) & Size & $\begin{array}{l}-0.010 \\
(0.699)\end{array}$ & $\begin{array}{l}-0.179 \\
(0.000)\end{array}$ & $\begin{array}{l}-0.020 \\
(0.444) \\
\end{array}$ & $\begin{array}{l}-0.093 \\
(0.000)\end{array}$ & $\begin{array}{l}0.020 \\
(0.458)\end{array}$ & $\begin{array}{l}0.035 \\
(0.183)\end{array}$ & $\begin{array}{l}0.345 \\
(0.000)\end{array}$ & $\begin{array}{l}0.328 \\
(0.000)\end{array}$ & $\begin{array}{l}0.114 \\
(0.000)\end{array}$ & $\begin{array}{l}0.016 \\
(0.554)\end{array}$ & 1 & & & & & & & \\
\hline (12) & Duality & $\begin{array}{l}0.066 \\
(0.012)\end{array}$ & $\begin{array}{l}0.183 \\
(0.000) \\
\end{array}$ & $\begin{array}{l}-0.104 \\
(0.000) \\
\end{array}$ & $\begin{array}{l}-0.055 \\
(0.037)\end{array}$ & $\begin{array}{l}0.039 \\
(0.139) \\
\end{array}$ & $\begin{array}{l}0.014 \\
(0.607) \\
\end{array}$ & $\begin{array}{c}0.021 \\
(0.430)\end{array}$ & $\begin{array}{l}-0.131 \\
(0.000)\end{array}$ & $\begin{array}{l}-0.079 \\
(0.002)\end{array}$ & $\begin{array}{c}0.189 \\
(0.000) \\
\end{array}$ & $\begin{array}{l}-0.153 \\
(0.000) \\
\end{array}$ & 1 & & & & & & \\
\hline (13) & Cash & $\begin{array}{l}0.022 \\
(0.412) \\
\end{array}$ & $\begin{array}{l}-0.010 \\
(0.698) \\
\end{array}$ & $\begin{array}{l}0.002 \\
(0.951) \\
\end{array}$ & $\begin{array}{l}-0.010 \\
(0.699) \\
\end{array}$ & $\begin{array}{l}-0.008 \\
(0.765) \\
\end{array}$ & $\begin{array}{l}-0.015 \\
(0.572) \\
\end{array}$ & $\begin{array}{l}0.015 \\
(0.564) \\
\end{array}$ & $\begin{array}{c}0.002 \\
(0.942) \\
\end{array}$ & $\begin{array}{l}-0.013 \\
(0.636) \\
\end{array}$ & $\begin{array}{l}0.024 \\
(0.368) \\
\end{array}$ & $\begin{array}{l}-0.091 \\
(0.000) \\
\end{array}$ & $\begin{array}{l}-0.036 \\
(0.179) \\
\end{array}$ & 1 & & & & & \\
\hline (14) & Bea & $\begin{array}{l}-0.049 \\
(0.062)\end{array}$ & $\begin{array}{l}-0.093 \\
(0.000)\end{array}$ & $\begin{array}{l}-0.178 \\
(0.000) \\
\end{array}$ & $\begin{array}{l}0.107 \\
(0.000)\end{array}$ & $\begin{array}{l}-0.119 \\
(0.000)\end{array}$ & $\begin{array}{l}0.049 \\
(0.063) \\
\end{array}$ & $\begin{array}{l}0.019 \\
(0.474)\end{array}$ & $\begin{array}{l}-0.128 \\
(0.000)\end{array}$ & $\begin{array}{l}-0.022 \\
(0.411)\end{array}$ & $\begin{array}{l}-0.009 \\
(0.729)\end{array}$ & $\begin{array}{l}0.099 \\
(0.000)\end{array}$ & $\begin{array}{l}0.034 \\
(0.198)\end{array}$ & $\begin{array}{l}-0.114 \\
(0.000)\end{array}$ & 1 & & & & \\
\hline (15) & Tang & $\begin{array}{l}-0.026 \\
(0.323)\end{array}$ & $\begin{array}{l}-0.078 \\
(0.003)\end{array}$ & $\begin{array}{l}0.183 \\
(0.000)\end{array}$ & $\begin{array}{l}0.017 \\
(0.522)\end{array}$ & $\begin{array}{l}-0.091 \\
(0.000)\end{array}$ & $\begin{array}{l}-0.108 \\
(0.000)\end{array}$ & $\begin{array}{l}0.030 \\
(0.264)\end{array}$ & $\begin{array}{c}0.031 \\
(0.235)\end{array}$ & $\begin{array}{l}0.056 \\
(0.035)\end{array}$ & $\begin{array}{l}-0.067 \\
(0.011)\end{array}$ & $\begin{array}{l}0.204 \\
(0.000)\end{array}$ & $\begin{array}{l}-0.186 \\
(0.000)\end{array}$ & $\begin{array}{l}-0.017 \\
(0.521)\end{array}$ & $\begin{array}{l}-0.198 \\
(0.000)\end{array}$ & 1 & & & \\
\hline (16) & Lev & $\begin{array}{l}-0.004 \\
(0.876) \\
\end{array}$ & $\begin{array}{l}-0.112 \\
(0.000) \\
\end{array}$ & $\begin{array}{l}-0.010 \\
(0.698) \\
\end{array}$ & $\begin{array}{l}-0.067 \\
(0.010) \\
\end{array}$ & $\begin{array}{l}-0.143 \\
(0.000) \\
\end{array}$ & $\begin{array}{l}0.009 \\
(0.743) \\
\end{array}$ & $\begin{array}{c}0.085 \\
(0.001) \\
\end{array}$ & $\begin{array}{l}-0.021 \\
(0.428) \\
\end{array}$ & $\begin{array}{l}-0.098 \\
(0.000) \\
\end{array}$ & $\begin{array}{l}-0.133 \\
(0.000) \\
\end{array}$ & $\begin{array}{l}0.375 \\
(0.000) \\
\end{array}$ & $\begin{array}{c}0.049 \\
(0.062) \\
\end{array}$ & $\begin{array}{l}-0.129 \\
(0.000) \\
\end{array}$ & $\begin{array}{c}0.499 \\
(0.000) \\
\end{array}$ & $\begin{array}{l}0.288 \\
(0.000) \\
\end{array}$ & 1 & & \\
\hline (17) & $\mathrm{MB}$ & $\begin{array}{l}0.015 \\
(0.570) \\
\end{array}$ & $\begin{array}{l}0.063 \\
(0.016) \\
\end{array}$ & $\begin{array}{l}-0.093 \\
(0.000) \\
\end{array}$ & $\begin{array}{l}-0.042 \\
(0.109) \\
\end{array}$ & $\begin{array}{c}0.067 \\
(0.010) \\
\end{array}$ & $\begin{array}{l}-0.013 \\
(0.615) \\
\end{array}$ & $\begin{array}{c}0.021 \\
(0.421) \\
\end{array}$ & $\begin{array}{c}0.142 \\
(0.000) \\
\end{array}$ & $\begin{array}{c}0.047 \\
(0.078) \\
\end{array}$ & $\begin{array}{c}0.091 \\
(0.000) \\
\end{array}$ & $\begin{array}{l}-0.013 \\
(0.637) \\
\end{array}$ & $\begin{array}{l}-0.094 \\
(0.000) \\
\end{array}$ & $\begin{array}{l}0.046 \\
(0.082) \\
\end{array}$ & $\begin{array}{l}-0.381 \\
(0.000) \\
\end{array}$ & $\begin{array}{l}-0.033 \\
(0.207) \\
\end{array}$ & $\begin{array}{l}-0.309 \\
(0.000) \\
\end{array}$ & 1 & \\
\hline (18) & Own & $\begin{array}{l}-0.008 \\
(0.770)\end{array}$ & $\begin{array}{l}0.008 \\
(0.765)\end{array}$ & $\begin{array}{l}-0.078 \\
(0.003)\end{array}$ & $\begin{array}{l}-0.006 \\
(0.796)\end{array}$ & $\begin{array}{l}-0.059 \\
(0.026)\end{array}$ & $\begin{array}{l}-0.017 \\
(0.534)\end{array}$ & $\begin{array}{l}-0.128 \\
(0.000)\end{array}$ & $\begin{array}{l}-0.212 \\
(0.000)\end{array}$ & $\begin{array}{c}-0.074 \\
(0.005)\end{array}$ & $\begin{array}{l}-0.152 \\
(0.000)\end{array}$ & $\begin{array}{l}-0.099 \\
(0.000)\end{array}$ & $\begin{array}{l}0.148 \\
(0.000)\end{array}$ & $\begin{array}{l}-0.037 \\
(0.161)\end{array}$ & $\begin{array}{l}0.099 \\
(0.000)\end{array}$ & $\begin{array}{l}0.159 \\
(0.000)\end{array}$ & $\begin{array}{l}0.263 \\
(0.000)\end{array}$ & $\begin{array}{l}-0.115 \\
(0.000)\end{array}$ & 1 \\
\hline
\end{tabular}


Table 4. Two-stage least squares (2SLS) results.

\begin{tabular}{|c|c|c|c|c|c|c|c|c|c|c|c|c|}
\hline & (1) & (2) & (3) & (4) & (5) & (6) & (7) & (8) & (9) & (10) & (11) & (12) \\
\hline & \multicolumn{4}{|c|}{ Individual Risk (IR) } & \multicolumn{4}{|c|}{ Systemic Risk (SR) } & \multicolumn{4}{|c|}{ Contribution Risk (CSR) } \\
\hline Senior $_{t-1}$ & $\begin{array}{c}-0.0039 \text { ** } \\
(0.052)\end{array}$ & & & & $\begin{array}{l}-0.0035 \\
(0.217) \\
\end{array}$ & & & & $\begin{array}{l}0.00034 \\
(0.778) \\
\end{array}$ & & & \\
\hline Nonsenior $_{t-1}$ & & $\begin{array}{c}-0.0071 * * * * \\
(0.000)\end{array}$ & & & & $\begin{array}{c}-0.0095 * * * \\
(0.000)\end{array}$ & & & & $\begin{array}{c}-0.0024 \text { ** } \\
(0.016)\end{array}$ & & \\
\hline$C E O_{t-1}$ & & & $\begin{array}{c}-0.0022 \text { ** } \\
(0.035)\end{array}$ & & & & $\begin{array}{c}-0.0027^{*} \\
(0.078)\end{array}$ & & & & $\begin{array}{l}-0.0004 \\
(0.483)\end{array}$ & \\
\hline Chair $_{t-1}$ & & & & $\begin{array}{c}-0.0030^{* * *} \\
(0.002)\end{array}$ & & & & $\begin{array}{c}-0.0039^{* * *} \\
(0.007)\end{array}$ & & & & $\begin{array}{l}-0.0008 \\
(0.178)\end{array}$ \\
\hline Manager & $\begin{array}{c}-0.0008 \\
(0.42)\end{array}$ & $\begin{array}{l}-0.0006 \\
(0.482)\end{array}$ & $\begin{array}{l}-0.0006 \\
(0.509)\end{array}$ & $\begin{array}{l}-0.0007 \\
(0.463)\end{array}$ & $\begin{array}{l}-0.0011 \\
(0.444)\end{array}$ & $\begin{array}{l}-0.0011 \\
(0.436)\end{array}$ & $\begin{array}{l}-0.0010 \\
(0.473)\end{array}$ & $\begin{array}{l}-0.0011 \\
(0.429)\end{array}$ & $\begin{array}{l}-0.0002 \\
(0.629)\end{array}$ & $\begin{array}{l}-0.0004 \\
(0.495)\end{array}$ & $\begin{array}{l}-0.0003 \\
(0.542)\end{array}$ & $\begin{array}{l}-0.0004 \\
(0.506)\end{array}$ \\
\hline Board & $\begin{array}{l}0.0002 \\
(0.898)\end{array}$ & $\begin{array}{l}0.0001 \\
(0.941)\end{array}$ & $\begin{array}{l}-0.0002 \\
(0.857)\end{array}$ & $\begin{array}{l}-0.0003 \\
(0.757)\end{array}$ & $\begin{array}{l}-0.0008 \\
(0.635)\end{array}$ & $\begin{array}{l}-0.0008 \\
(0.636)\end{array}$ & $\begin{array}{l}-0.0012 \\
(0.485)\end{array}$ & $\begin{array}{l}-0.0014 \\
(0.415)\end{array}$ & $\begin{array}{l}-0.0010 \\
(0.181)\end{array}$ & $\begin{array}{l}-0.0009 \\
(0.215)\end{array}$ & $\begin{array}{l}-0.0010 \\
(0.175)\end{array}$ & $\begin{array}{l}-0.0010 \\
(0.155)\end{array}$ \\
\hline Achair & $\begin{array}{l}0.0007 \\
(0.728)\end{array}$ & $\begin{array}{l}0.0007 \\
(0.697)\end{array}$ & $\begin{array}{l}0.0012 \\
(0.520)\end{array}$ & $\begin{array}{l}0.0011 \\
(0.572)\end{array}$ & $\begin{array}{l}0.0026 \\
(0.360)\end{array}$ & $\begin{array}{l}0.0024 \\
(0.392)\end{array}$ & $\begin{array}{l}0.0030 \\
(0.271) \\
\end{array}$ & $\begin{array}{l}0.0028 \\
(0.304)\end{array}$ & $\begin{array}{l}0.0019 \\
(0.110)\end{array}$ & $\begin{array}{l}0.0016 \\
(0.166)\end{array}$ & $\begin{array}{l}0.0018 \\
(0.122)\end{array}$ & $\begin{array}{l}0.0018 \\
(0.133)\end{array}$ \\
\hline Aceo & $\begin{array}{l}-0.0025 \\
(0.172)\end{array}$ & $\begin{array}{l}-0.0025 \\
(0.170)\end{array}$ & $\begin{array}{l}-0.0026 \\
(0.168)\end{array}$ & $\begin{array}{l}-0.0026 \\
(0.159)\end{array}$ & $\begin{array}{l}-0.0037 \\
(0.166)\end{array}$ & $\begin{array}{l}-0.0038 \\
(0.150)\end{array}$ & $\begin{array}{l}-0.0038 \\
(0.153)\end{array}$ & $\begin{array}{l}-0.0039 \\
(0.143)\end{array}$ & $\begin{array}{l}-0.0011 \\
(0.303)\end{array}$ & $\begin{array}{l}-0.0013 \\
(0.252)\end{array}$ & $\begin{array}{l}-0.0012 \\
(0.267)\end{array}$ & $\begin{array}{l}-0.0013 \\
(0.254)\end{array}$ \\
\hline Size & $\begin{array}{l}-0.0022 \\
(0.000)\end{array}$ & $\begin{array}{l}-0.0023 \\
(0.000)\end{array}$ & $\begin{array}{l}-0.0021 \\
(0.000)\end{array}$ & $\begin{array}{l}-0.0021 \\
(0.000)\end{array}$ & $\begin{array}{l}-0.0027 \\
(0.000)\end{array}$ & $\begin{array}{c}-0.0029 \\
(0.000)\end{array}$ & $\begin{array}{l}-0.0027 \\
(0.000)\end{array}$ & $\begin{array}{l}-0.0027 \\
(0.000)\end{array}$ & $\begin{array}{l}-0.0005 \\
(0.001)\end{array}$ & $\begin{array}{l}-0.0006 \\
(0.000)\end{array}$ & $\begin{array}{l}-0.0005 \\
(0.002)\end{array}$ & $\begin{array}{l}-0.0005 \\
(0.002) \\
\end{array}$ \\
\hline Duality & $\begin{array}{l}0.0007 \\
(0.437)\end{array}$ & $\begin{array}{l}0.0011 \\
(0.200)\end{array}$ & $\begin{array}{l}0.0007 \\
(0.437)\end{array}$ & $\begin{array}{l}0.0007 \\
(0.443)\end{array}$ & $\begin{array}{l}0.0006 \\
(0.619)\end{array}$ & $\begin{array}{l}0.0013 \\
(0.307)\end{array}$ & $\begin{array}{l}0.0007 \\
(0.594)\end{array}$ & $\begin{array}{l}0.0007 \\
(0.595)\end{array}$ & $\begin{array}{l}-0.0000 \\
(0.916)\end{array}$ & $\begin{array}{l}0.0002 \\
(0.760)\end{array}$ & $\begin{array}{l}-0.0000 \\
(0.985)\end{array}$ & $\begin{array}{l}-0.0000 \\
(0.995)\end{array}$ \\
\hline Cash & $\begin{array}{l}0.0018 \\
(0.520)\end{array}$ & $\begin{array}{l}0.0018 \\
(0.532)\end{array}$ & $\begin{array}{l}0.0017 \\
(0.544)\end{array}$ & $\begin{array}{l}0.0018 \\
(0.536)\end{array}$ & $\begin{array}{l}0.0030 \\
(0.469)\end{array}$ & $\begin{array}{l}0.0029 \\
(0.478)\end{array}$ & $\begin{array}{l}0.0029 \\
(0.487)\end{array}$ & $\begin{array}{l}0.0029 \\
(0.482)\end{array}$ & $\begin{array}{l}0.0011 \\
(0.512)\end{array}$ & $\begin{array}{l}0.0011 \\
(0.518)\end{array}$ & $\begin{array}{l}0.0011 \\
(0.519)\end{array}$ & $\begin{array}{l}0.0011 \\
(0.518)\end{array}$ \\
\hline Bea & $\begin{array}{l}0.0051 \\
(0.015)\end{array}$ & $\begin{array}{l}0.0049 \\
(0.018)\end{array}$ & $\begin{array}{l}0.0048 \\
(0.021)\end{array}$ & $\begin{array}{l}0.0052 \\
(0.012)\end{array}$ & $\begin{array}{l}0.0079 \\
(0.008)\end{array}$ & $\begin{array}{l}0.0076 \\
(0.011)\end{array}$ & $\begin{array}{l}0.0076 \\
(0.012)\end{array}$ & $\begin{array}{l}0.0081 \\
(0.007)\end{array}$ & $\begin{array}{l}0.0028 \\
(0.026)\end{array}$ & $\begin{array}{l}0.0027 \\
(0.030)\end{array}$ & $\begin{array}{l}0.0027 \\
(0.03)\end{array}$ & $\begin{array}{l}0.0028 \\
(0.025)\end{array}$ \\
\hline Tang & $\begin{array}{l}0.0042 \\
(0.043) \\
\end{array}$ & $\begin{array}{l}0.0041 \\
(0.045) \\
\end{array}$ & $\begin{array}{l}0.0039 \\
(0.062) \\
\end{array}$ & $\begin{array}{l}0.0036 \\
(0.085) \\
\end{array}$ & $\begin{array}{l}0.0071 \\
(0.017) \\
\end{array}$ & $\begin{array}{l}0.0071 \\
(0.017) \\
\end{array}$ & $\begin{array}{l}0.0067 \\
(0.024)\end{array}$ & $\begin{array}{l}0.0064 \\
(0.033)\end{array}$ & $\begin{array}{l}0.0029 \\
(0.020)\end{array}$ & $\begin{array}{l}0.0029 \\
(0.020)\end{array}$ & $\begin{array}{l}0.0029 \\
(0.022)\end{array}$ & $\begin{array}{l}0.0028 \\
(0.028)\end{array}$ \\
\hline Lev & $\begin{array}{l}0.0021 \\
(0.421)\end{array}$ & $\begin{array}{l}0.0019 \\
(0.476)\end{array}$ & $\begin{array}{l}0.0017 \\
(0.525)\end{array}$ & $\begin{array}{l}0.0024 \\
(0.358)\end{array}$ & $\begin{array}{l}0.0012 \\
(0.757)\end{array}$ & $\begin{array}{l}0.0009 \\
(0.814)\end{array}$ & $\begin{array}{l}0.0007 \\
(0.859)\end{array}$ & $\begin{array}{l}0.0016 \\
(0.672)\end{array}$ & $\begin{array}{l}-0.0009 \\
(0.550)\end{array}$ & $\begin{array}{l}-0.0009 \\
(0.538)\end{array}$ & $\begin{array}{c}-0.0010 \\
(0.53)\end{array}$ & $\begin{array}{c}-0.0008 \\
(0.606)\end{array}$ \\
\hline MB & $\begin{array}{c}-0.0000 \\
(0.278)\end{array}$ & $\begin{array}{c}-0.0000 \\
(0.332)\end{array}$ & $\begin{array}{l}-0.0000 \\
(0.277)\end{array}$ & $\begin{array}{c}-0.0000 \\
(0.269)\end{array}$ & $\begin{array}{l}-0.0000 \\
(0.407)\end{array}$ & $\begin{array}{c}-0.0000 \\
(0.473)\end{array}$ & $\begin{array}{c}-0.0000 \\
(0.407)\end{array}$ & $\begin{array}{c}-0.0000 \\
(0.398)\end{array}$ & $\begin{array}{c}-0.0000 \\
(0.863)\end{array}$ & $\begin{array}{c}-0.0000 \\
(0.920)\end{array}$ & $\begin{array}{c}-0.0000 \\
(0.865)\end{array}$ & $\begin{array}{c}-0.0000 \\
(0.860)\end{array}$ \\
\hline Own & $\begin{array}{l}-0.0032 \\
(0.545)\end{array}$ & $\begin{array}{c}-0.0036 \\
(0.497)\end{array}$ & $\begin{array}{l}-0.0028 \\
(0.591)\end{array}$ & $\begin{array}{l}-0.0038 \\
(0.472)\end{array}$ & $\begin{array}{c}-0.0059 \\
(0.437)\end{array}$ & $\begin{array}{l}-0.0068 \\
(0.366)\end{array}$ & $\begin{array}{c}-0.0058 \\
(0.449)\end{array}$ & $\begin{array}{l}-0.0071 \\
(0.357)\end{array}$ & $\begin{array}{l}-0.0027 \\
(0.400)\end{array}$ & $\begin{array}{c}-0.0032 \\
(0.309)\end{array}$ & $\begin{array}{c}-0.0029 \\
(0.364)\end{array}$ & $\begin{array}{l}-0.0032 \\
(0.319)\end{array}$ \\
\hline Constants & $\begin{array}{l}0.0824 \\
(0.000)\end{array}$ & $\begin{array}{l}0.0866 \\
(0.000)\end{array}$ & $\begin{array}{l}0.0790 \\
(0.000)\end{array}$ & $\begin{array}{l}0.0810 \\
(0.000) \\
\end{array}$ & $\begin{array}{l}0.1005 \\
(0.000) \\
\end{array}$ & $\begin{array}{l}0.1084 \\
(0.000) \\
\end{array}$ & $\begin{array}{l}0.0980 \\
(0.000) \\
\end{array}$ & $\begin{array}{l}0.1006 \\
(0.000)\end{array}$ & $\begin{array}{l}0.0181 \\
(0.014) \\
\end{array}$ & $\begin{array}{l}0.0218 \\
(0.003) \\
\end{array}$ & $\begin{array}{l}0.0189 \\
(0.01) \\
\end{array}$ & $\begin{array}{l}0.0196 \\
(0.008)\end{array}$ \\
\hline Obs & 1197 & 1197 & 1197 & 1197 & 1197 & 1197 & 1197 & 1197 & 1197 & 1197 & 1197 & 1197 \\
\hline W-Chi2 & 0.000 & 0.000 & 0.000 & 0.000 & 0.000 & 0.000 & 0.000 & 0.000 & 0.000 & 0.000 & 0.000 & 0.000 \\
\hline R-squared & 0.0671 & 0.0764 & 0.0693 & 0.0683 & 0.0504 & 0.0583 & 0.0521 & 0.0507 & 0.0152 & 0.0159 & 0.0140 & 0.0129 \\
\hline Sargan & 0.7663 & 0.8396 & 0.4100 & 0.7255 & 0.9738 & 0.9921 & 0.5505 & 0.8680 & 0.6791 & 0.7214 & 0.9564 & 0.8527 \\
\hline Endogen & 0.0539 & 0.0432 & 0.0670 & 0.0440 & 0.0463 & 0.0322 & 0.0510 & 0.0333 & 0.1260 & 0.0853 & 0.1119 & 0.0936 \\
\hline
\end{tabular}

This table presents 2SLS regressions with robust standard errors clustered by firms where ownership is instrumented with the female on board concentration of other companies of the same year and with the logarithm of the firm's age. Sargan presents the $p$-value for the test of over-identifying restrictions with the null of the validity of the instruments. Endogen presents the $p$-values of the Wooldridge score test of endogeneity with the null hypothesis if the exogeneity of the variables. The Wald chi2 presents the $p$-values for the model validity. T statistics in parentheses. ${ }^{*} p<0.1 ; * * p<0.05 ; * * * 00.01$ 


\subsection{Generalized Method of Moments (GMM)}

Table 5 shows whether the impact of female managers on corporate risk-taking is robust to the application of the generalized method of moments (GMM) model. The outcomes are still remained as 2SLS's results and corroborate our main findings to support $\mathbf{H} \mathbf{2} \mathbf{a}$ and $\mathbf{H} \mathbf{2} \mathbf{b}$. These results confirm that the representation of women as the firm's CEO and Chairman reduce both individual risk (IR) and systemic risk (SR). In particular, women are mentioned as less risky [53], and more conservative than male counterparts $[52,69]$. Thus, the negative relationship between top female leaders infirm and corporate risk-taking is established. Furthermore, we found no significant effect of female CEO to CSR, which reject $\mathbf{H} 2 \mathbf{c}$.

In addition, to test $\mathbf{H 1 a}, \mathbf{H 1 b}$, and $\mathbf{H 1 c}$, we conjecture that the risk orientation of a firm is heterogeneous and differs depending on the managing levels. We find that by distinguishing senior and non-senior managers, all risk attributes (including IR, SR, and CSR) mainly decrease by the increase in the female non-senior managers, whereas senior managers reduce only individual risk (IR). Thus, these findings only support H1a. These findings are consistent with [1,11], which encourage the high proportion of women in managerial levels to reduce downside risk. In another hand, the linkage between the number of female managers and executives is probably from the outside pressure to create greater diversity, instead of firm value enhancing [81]. In that context, if women are not required to increase the firm's benefits, female managers and executives are more associated with less aggressive investment policies and better acquisition. Therefore, the role of women in firm management is more consistent with institutional ownership rather than their interest in maximizing the managerial incentives. Thus, that characteristic reduces excessive risk-taking behavior which decreases the firm's downside risk, especially in the manufacturing sector [82].

\subsection{Further Analysis}

To further our analysis, we continue running the 2SLS and GMM to estimate whether the female executive or non-executive makes the impact to firm's downside risk. After running the robustness tests for endogeneity concern, the outcomes from Table 6 indicate that the women make the significant effect on individual risk only in the case of executive director. That finding acknowledge that the women benefit firms in higher power position such as executive directors or managers [33]. In addition, we find no significant relationship between female non-executive directors and risk attributes. That result clarifies the findings above that the proportion of female senior managers reduces firm risk-taking only if they hold a more powerful position in board of directors. In another meaning, it confirms that women need to face a "second glass ceiling" since their power is limited by non-executive even after attending to the board level [32]. In line with that view, the role of a non-executive director is commonly mentioned as a board supervisor that scrutinizes executive director's performance, information asymmetries and dysfunctional working relation rather than involvement to a firm's strategy planning [83].

In the context of gender diversity in high or low-risk firms, Table 7 shows the quantile regression to consider heterogeneous responses to gender diversity in executive management. Intuitively, high and low-risk firms (Q25: 25th percentile and Q75: 75th percentile) might respond differently from the women on alternative management levels. This viewpoint implies that it is possible to represent the relationship between gender diversity and risk with different estimation according to the firms' riskiness. We justify the use of quantile regressions with the Breusch-Pagan/Cool Weisberg tests which reject the null hypothesis if homoscedastic errors for all models. We find that in high-risk firms (Q75), the appearance of women in non-senior management levels and female chairman decreases all risk attributes. Furthermore, in low-risk firms (Q25), the outcomes indicate that the appearance of women in both senior and non-senior management plays a critical role in reducing firm risk-taking. In addition, the firm ownership increases the risk-taking in case of high-risk firms which explained the role of power concentration incorporates the risk. 
Table 5. Generalized method of moments (GMM) results.

\begin{tabular}{|c|c|c|c|c|c|c|c|c|c|c|c|c|}
\hline & (1) & (2) & (3) & (4) & (5) & (6) & (7) & (8) & (9) & (10) & (11) & (12) \\
\hline & \multicolumn{4}{|c|}{ Individual Risk (IR) } & \multicolumn{4}{|c|}{ Systemic Risk (SR) } & \multicolumn{4}{|c|}{ Contribution Risk (CSR) } \\
\hline Senior $_{t-1}$ & $\begin{array}{c}-0.0039 * * \\
(0.046)\end{array}$ & & & & $\begin{array}{l}-0.0035 \\
(0.185)\end{array}$ & & & & $\begin{array}{l}0.00010 \\
(0.904)\end{array}$ & & & \\
\hline Nonsenior $_{t-1}$ & & $\begin{array}{c}-0.0071 \text { *** } \\
(0.000)\end{array}$ & & & & $\begin{array}{c}-0.0095 \text { *** } \\
(0.000)\end{array}$ & & & & $\begin{array}{c}-0.0021 \text { ** } \\
(0.036)\end{array}$ & & \\
\hline$C E O_{t-1}$ & & & $\begin{array}{c}-0.0022 \text { ** } \\
(0.013)\end{array}$ & & & & $\begin{array}{c}-0.0027 \text { ** } \\
(0.032)\end{array}$ & & & & $\begin{array}{l}-0.0004 \\
(0.293)\end{array}$ & \\
\hline Chair $_{t-1}$ & & & & $\begin{array}{c}-0.0031^{* * *} \\
(0.002)\end{array}$ & & & & $\begin{array}{c}-0.0039 * * * \\
(0.002)\end{array}$ & & & & $\begin{array}{c}-0.0008 * * * * \\
(0.012)\end{array}$ \\
\hline Manager & $\begin{array}{l}-0.0007 \\
(0.402)\end{array}$ & $\begin{array}{l}-0.0007 \\
(0.416)\end{array}$ & $\begin{array}{l}-0.0008 \\
(0.364)\end{array}$ & $\begin{array}{l}-0.0008 \\
(0.375)\end{array}$ & $\begin{array}{l}-0.0011 \\
(0.373)\end{array}$ & $\begin{array}{l}-0.0011 \\
(0.368)\end{array}$ & $\begin{array}{l}-0.0013 \\
(0.294)\end{array}$ & $\begin{array}{l}-0.0012 \\
(0.325)\end{array}$ & $\begin{array}{l}-0.0004 \\
(0.294)\end{array}$ & $\begin{array}{l}-0.0002 \\
(0.449)\end{array}$ & $\begin{array}{l}-0.0003 \\
(0.284)\end{array}$ & $\begin{array}{l}-0.0003 \\
(0.374)\end{array}$ \\
\hline Board & $\begin{array}{l}0.0002 \\
(0.889)\end{array}$ & $\begin{array}{l}0.0001 \\
(0.928)\end{array}$ & $\begin{array}{l}-0.0002 \\
(0.857)\end{array}$ & $\begin{array}{l}-0.0003 \\
(0.749)\end{array}$ & $\begin{array}{l}-0.0008 \\
(0.614)\end{array}$ & $\begin{array}{l}-0.0008 \\
(0.613)\end{array}$ & $\begin{array}{l}-0.0012 \\
(0.463)\end{array}$ & $\begin{array}{l}-0.0014 \\
(0.392)\end{array}$ & $\begin{array}{l}-0.0006 \\
(0.311)\end{array}$ & $\begin{array}{l}-0.0008 \\
(0.295)\end{array}$ & $\begin{array}{l}-0.0010 \\
(0.221)\end{array}$ & $\begin{array}{l}-0.0010 \\
(0.209)\end{array}$ \\
\hline Achair & $\begin{array}{l}0.0007 \\
(0.748)\end{array}$ & $\begin{array}{l}0.0008 \\
(0.715)\end{array}$ & $\begin{array}{l}0.0013 \\
(0.538)\end{array}$ & $\begin{array}{l}0.0011 \\
(0.592)\end{array}$ & $\begin{array}{l}0.0026 \\
(0.401)\end{array}$ & $\begin{array}{l}0.0024 \\
(0.422)\end{array}$ & $\begin{array}{l}0.0031 \\
(0.297)\end{array}$ & $\begin{array}{l}0.0029 \\
(0.333)\end{array}$ & $\begin{array}{l}0.0015 \\
(0.181)\end{array}$ & $\begin{array}{l}0.0015 \\
(0.182)\end{array}$ & $\begin{array}{l}0.0018 \\
(0.127)\end{array}$ & $\begin{array}{l}0.0017 \\
(0.147)\end{array}$ \\
\hline Aceo & $\begin{array}{l}-0.0026 \\
(0.172)\end{array}$ & $\begin{array}{l}-0.0025 \\
(0.186)\end{array}$ & $\begin{array}{l}-0.0024 \\
(0.212)\end{array}$ & $\begin{array}{l}-0.0025 \\
(0.178)\end{array}$ & $\begin{array}{l}-0.0037 \\
(0.171)\end{array}$ & $\begin{array}{l}-0.0038 \\
(0.166)\end{array}$ & $\begin{array}{l}-0.0036 \\
(0.195)\end{array}$ & $\begin{array}{l}-0.0039 \\
(0.163)\end{array}$ & $\begin{array}{l}-0.0009 \\
(0.333)\end{array}$ & $\begin{array}{l}-0.0013 \\
(0.224)\end{array}$ & $\begin{array}{l}-0.0012 \\
(0.242)\end{array}$ & $\begin{array}{l}-0.0013 \\
(0.218)\end{array}$ \\
\hline Size & $\begin{array}{l}-0.0021 \\
(0.000)\end{array}$ & $\begin{array}{l}-0.0023 \\
(0.000)\end{array}$ & $\begin{array}{l}-0.0021 \\
(0.000)\end{array}$ & $\begin{array}{l}-0.0021 \\
(0.000)\end{array}$ & $\begin{array}{l}-0.0027 \\
(0.000)\end{array}$ & $\begin{array}{l}-0.0029 \\
(0.000)\end{array}$ & $\begin{array}{l}-0.0027 \\
(0.000)\end{array}$ & $\begin{array}{l}-0.0027 \\
(0.000)\end{array}$ & $\begin{array}{l}-0.0005 \\
(0.000)\end{array}$ & $\begin{array}{l}-0.0006 \\
(0.000)\end{array}$ & $\begin{array}{l}-0.0005 \\
(0.000)\end{array}$ & $\begin{array}{l}-0.0005 \\
(0.000)\end{array}$ \\
\hline Duality & $\begin{array}{l}0.0007 \\
(0.408)\end{array}$ & $\begin{array}{l}0.0011 \\
(0.18)\end{array}$ & $\begin{array}{l}0.0006 \\
(0.437)\end{array}$ & $\begin{array}{l}0.0007 \\
(0.431)\end{array}$ & $\begin{array}{l}0.0006 \\
(0.592)\end{array}$ & $\begin{array}{l}0.0013 \\
(0.255)\end{array}$ & $\begin{array}{l}0.0006 \\
(0.573)\end{array}$ & $\begin{array}{l}0.0007 \\
(0.568)\end{array}$ & $\begin{array}{l}0.0000 \\
(0.923)\end{array}$ & $\begin{array}{l}0.0002 \\
(0.641)\end{array}$ & $\begin{array}{l}-0.0000 \\
(0.982)\end{array}$ & $\begin{array}{l}-0.0000 \\
(0.999)\end{array}$ \\
\hline Cash & $\begin{array}{l}0.0019 \\
(0.53)\end{array}$ & $\begin{array}{l}0.0017 \\
(0.578)\end{array}$ & $\begin{array}{l}0.0014 \\
(0.66)\end{array}$ & $\begin{array}{l}0.0016 \\
(0.592)\end{array}$ & $\begin{array}{l}0.0030 \\
(0.468)\end{array}$ & $\begin{array}{l}0.0029 \\
(0.475)\end{array}$ & $\begin{array}{l}0.0023 \\
(0.576)\end{array}$ & $\begin{array}{l}0.0028 \\
(0.497)\end{array}$ & $\begin{array}{l}0.0010 \\
(0.39)\end{array}$ & $\begin{array}{l}0.0013 \\
(0.246)\end{array}$ & $\begin{array}{l}0.0011 \\
(0.349)\end{array}$ & $\begin{array}{l}0.0012 \\
(0.288)\end{array}$ \\
\hline Bea & $\begin{array}{l}0.0052 \\
(0.014)\end{array}$ & $\begin{array}{l}0.0049 \\
(0.02)\end{array}$ & $\begin{array}{l}0.0047 \\
(0.027)\end{array}$ & $\begin{array}{l}0.0052 \\
(0.014)\end{array}$ & $\begin{array}{l}0.0079 \\
(0.005)\end{array}$ & $\begin{array}{l}0.0076 \\
(0.007)\end{array}$ & $\begin{array}{l}0.0073 \\
(0.011)\end{array}$ & $\begin{array}{l}0.0080 \\
(0.005)\end{array}$ & $\begin{array}{l}0.0024 \\
(0.005)\end{array}$ & $\begin{array}{l}0.0028 \\
(0.010)\end{array}$ & $\begin{array}{l}0.0027 \\
(0.010)\end{array}$ & $\begin{array}{l}0.0029 \\
(0.007)\end{array}$ \\
\hline Tang & $\begin{array}{l}0.0042 \\
(0.072)\end{array}$ & $\begin{array}{l}0.0042 \\
(0.069)\end{array}$ & $\begin{array}{l}0.0040 \\
(0.081)\end{array}$ & $\begin{array}{l}0.0036 \\
(0.115)\end{array}$ & $\begin{array}{l}0.0071 \\
(0.045)\end{array}$ & $\begin{array}{l}0.0071 \\
(0.044)\end{array}$ & $\begin{array}{l}0.0071 \\
(0.044)\end{array}$ & $\begin{array}{l}0.0064 \\
(0.066)\end{array}$ & $\begin{array}{l}0.0027 \\
(0.053)\end{array}$ & $\begin{array}{l}0.0027 \\
(0.050)\end{array}$ & $\begin{array}{l}0.0029 \\
(0.034)\end{array}$ & $\begin{array}{l}0.0026 \\
(0.053)\end{array}$ \\
\hline Lev & $\begin{array}{l}0.0020 \\
(0.412)\end{array}$ & $\begin{array}{l}0.0019 \\
(0.429)\end{array}$ & $\begin{array}{l}0.0020 \\
(0.423)\end{array}$ & $\begin{array}{l}0.0026 \\
(0.298)\end{array}$ & $\begin{array}{l}0.0011 \\
(0.749) \\
\end{array}$ & $\begin{array}{l}0.0009 \\
(0.807)\end{array}$ & $\begin{array}{l}0.0009 \\
(0.797)\end{array}$ & $\begin{array}{l}0.0017 \\
(0.643)\end{array}$ & $\begin{array}{l}-0.0003 \\
(0.821)\end{array}$ & $\begin{array}{l}-0.0007 \\
(0.677)\end{array}$ & $\begin{array}{l}-0.0010 \\
(0.577)\end{array}$ & $\begin{array}{l}-0.0008 \\
(0.651)\end{array}$ \\
\hline MB & $\begin{array}{l}-0.0000 \\
(0.296)\end{array}$ & $\begin{array}{l}-0.0000 \\
(0.344)\end{array}$ & $\begin{array}{l}-0.0000 \\
(0.264)\end{array}$ & $\begin{array}{c}-0.0000 \\
(0.264)\end{array}$ & $\begin{array}{l}-0.0000 \\
(0.353)\end{array}$ & $\begin{array}{c}-0.0000 \\
(0.42)\end{array}$ & $\begin{array}{c}-0.0000 \\
(0.31)\end{array}$ & $\begin{array}{c}-0.0000 \\
(0.323)\end{array}$ & $\begin{array}{l}-0.0000 \\
(0.684)\end{array}$ & $\begin{array}{l}-0.0000 \\
(0.966)\end{array}$ & $\begin{array}{c}-0.0000 \\
(0.693)\end{array}$ & $\begin{array}{l}-0.0000 \\
(0.777)\end{array}$ \\
\hline Own & $\begin{array}{l}-0.0031 \\
(0.532)\end{array}$ & $\begin{array}{l}-0.0036 \\
(0.471)\end{array}$ & $\begin{array}{l}-0.0031 \\
(0.538)\end{array}$ & $\begin{array}{l}-0.0039 \\
(0.441)\end{array}$ & $\begin{array}{c}-0.0059 \\
(0.419)\end{array}$ & $\begin{array}{l}-0.0068 \\
(0.344)\end{array}$ & $\begin{array}{l}-0.0062 \\
(0.399)\end{array}$ & $\begin{array}{c}-0.0071 \\
(0.33)\end{array}$ & $\begin{array}{l}-0.0033 \\
(0.255)\end{array}$ & $\begin{array}{l}-0.0034 \\
(0.246)\end{array}$ & $\begin{array}{l}-0.0029 \\
(0.332)\end{array}$ & $\begin{array}{l}-0.0031 \\
(0.294)\end{array}$ \\
\hline Constants & \begin{tabular}{|l}
0.0825 \\
$(0.000)$ \\
\end{tabular} & $\begin{array}{l}0.0865 \\
(0.000)\end{array}$ & $\begin{array}{l}0.0789 \\
(0.000) \\
\end{array}$ & $\begin{array}{l}0.0809 \\
(0.000)\end{array}$ & $\begin{array}{l}0.1005 \\
(0.000) \\
\end{array}$ & $\begin{array}{l}0.1084 \\
(0.000) \\
\end{array}$ & $\begin{array}{l}0.0979 \\
(0.000) \\
\end{array}$ & $\begin{array}{l}0.1005 \\
(0.000) \\
\end{array}$ & $\begin{array}{l}0.0188 \\
(0.000)\end{array}$ & $\begin{array}{l}.0218 \\
(0.000)\end{array}$ & $\begin{array}{l}.0189 \\
(0.000)\end{array}$ & $\begin{array}{l}0.0197 \\
(0.000)\end{array}$ \\
\hline Obs & 1197 & 1197 & 1197 & 1197 & 1197 & 1197 & 1197 & 1197 & 1197 & 1197 & 1197 & 1197 \\
\hline R-squared & 0.0674 & 0.0762 & 0.0678 & 0.0677 & 0.0504 & 0.0583 & 0.0505 & 0.0503 & 0.0098 & 0.0145 & 0.0138 & 0.0132 \\
\hline Hansen' J & 0.7535 & 0.8413 & 0.4055 & 0.7270 & 0.9700 & 0.9915 & 0.5024 & 0.8543 & 0.5487 & 0.5897 & 0.9241 & 0.7506 \\
\hline
\end{tabular}

This table presents one-step GMM estimations by firms where ownership is instrumented with the female on board concentration of other companies of the same year and with the logarithm of the firm's age. Hansen' J presents the $p$-value for the test of over-identifying restrictions with the null of the validity of the instruments. ${ }^{* *} p<0.05$; ${ }^{* * *} p<0.01$. 
Table 6. The impact of female executive and non-executive directors onboard and downside risk.

\begin{tabular}{|c|c|c|c|c|c|c|c|c|c|c|c|c|}
\hline & IR (1) & SR (2) & CR (3) & IR (4) & SR (5) & CR (6) & IR (7) & SR (8) & CR (9) & IR (10) & SR (11) & CR (12) \\
\hline & \multicolumn{6}{|c|}{ 2SLS } & \multicolumn{6}{|c|}{ GMM } \\
\hline$E x e_{t-1}$ & & & & $\begin{array}{c}-0.0043 * \\
(0.074)\end{array}$ & $\begin{array}{c}-0.0053 \\
(0.127)\end{array}$ & $\begin{array}{c}-0.0009 \\
(0.503)\end{array}$ & & & & $\begin{array}{c}-0.0043 * \\
(0.086)\end{array}$ & $\begin{array}{c}-0.0053 \\
(0.115)\end{array}$ & $\begin{array}{c}-0.0008 \\
(0.436)\end{array}$ \\
\hline Nonexe $_{t-1}$ & $\begin{array}{c}-0.0030 \\
(0.423)\end{array}$ & $\begin{array}{l}0.0001 \\
(0.976) \\
\end{array}$ & $\begin{array}{l}0.0029 \\
(0.163) \\
\end{array}$ & & & & $\begin{array}{c}-0.0028 \\
(0.413)\end{array}$ & $\begin{array}{l}-0.0012 \\
(0.836)\end{array}$ & $\begin{array}{l}0.0006 \\
(0.820) \\
\end{array}$ & & & \\
\hline Manager & $\begin{array}{c}-0.0006 \\
(0.522)\end{array}$ & $\begin{array}{l}-0.0007 \\
(0.597)\end{array}$ & $\begin{array}{l}-0.0001 \\
(0.844)\end{array}$ & $\begin{array}{c}-0.0005 \\
(0.591)\end{array}$ & $\begin{array}{c}-0.0008 \\
(0.537)\end{array}$ & $\begin{array}{c}-0.0003 \\
(0.564)\end{array}$ & $\begin{array}{c}-0.0007 \\
(0.401)\end{array}$ & $\begin{array}{c}-0.0010 \\
(0.382)\end{array}$ & $\begin{array}{c}-0.0003 \\
(0.281)\end{array}$ & $\begin{array}{c}-0.0005 \\
(0.525)\end{array}$ & $\begin{array}{c}-0.0008 \\
(0.465)\end{array}$ & $\begin{array}{c}-0.0002 \\
(0.469)\end{array}$ \\
\hline Board & $\begin{array}{l}0.0001 \\
(0.955)\end{array}$ & $\begin{array}{l}-0.0011 \\
(0.535)\end{array}$ & $\begin{array}{l}-0.0011 \\
(0.118)\end{array}$ & $\begin{array}{c}-0.0000 \\
(0.946)\end{array}$ & $\begin{array}{c}-0.0010 \\
(0.544)\end{array}$ & $\begin{array}{c}-0.0010 \\
(0.184)\end{array}$ & $\begin{array}{l}0.0001 \\
(0.954)\end{array}$ & $\begin{array}{c}-0.0007 \\
(0.680)\end{array}$ & $\begin{array}{c}-0.0004 \\
(0.487)\end{array}$ & $\begin{array}{c}-0.0000 \\
(0.947)\end{array}$ & $\begin{array}{c}-0.0010 \\
(0.522)\end{array}$ & $\begin{array}{c}-0.0009 \\
(0.250)\end{array}$ \\
\hline Achair & $\begin{array}{l}0.0010 \\
(0.616)\end{array}$ & $\begin{array}{l}0.0032 \\
(0.256)\end{array}$ & $\begin{array}{l}0.0022 \\
(0.062)\end{array}$ & $\begin{array}{l}0.0012 \\
(0.529)\end{array}$ & $\begin{array}{l}0.0030 \\
(0.277)\end{array}$ & $\begin{array}{l}0.0018 \\
(0.124)\end{array}$ & $\begin{array}{l}0.0010 \\
(0.629)\end{array}$ & $\begin{array}{c}0.00286 \\
(0.373)\end{array}$ & $\begin{array}{l}0.00142 \\
(0.250)\end{array}$ & $\begin{array}{l}0.0012 \\
(0.561)\end{array}$ & $\begin{array}{l}0.0030 \\
(0.316)\end{array}$ & $\begin{array}{l}0.0018 \\
(0.139)\end{array}$ \\
\hline Aceo & $\begin{array}{c}-0.0023 \\
(0.221) \\
\end{array}$ & $\begin{array}{c}-0.0035 \\
(0.193) \\
\end{array}$ & $\begin{array}{c}-0.0012 \\
(0.286) \\
\end{array}$ & $\begin{array}{c}-0.0025 \\
(0.17) \\
\end{array}$ & $\begin{array}{c}-0.0038 \\
(0.153) \\
\end{array}$ & $\begin{array}{c}-0.0012 \\
(0.261) \\
\end{array}$ & $\begin{array}{c}-0.0022 \\
(0.258) \\
\end{array}$ & $\begin{array}{c}-0.0033 \\
(0.242) \\
\end{array}$ & $\begin{array}{c}-0.0011 \\
(0.302) \\
\end{array}$ & $\begin{array}{c}-0.0025 \\
(0.187) \\
\end{array}$ & $\begin{array}{c}-0.0038 \\
(0.160) \\
\end{array}$ & $\begin{array}{c}-0.0013 \\
(0.176) \\
\end{array}$ \\
\hline Size & $\begin{array}{l}-0.0021 \\
(0.000)\end{array}$ & $\begin{array}{c}-0.0028 \\
(0.000)\end{array}$ & $\begin{array}{l}-0.0006 \\
(0.001)\end{array}$ & $\begin{array}{c}-0.0022 \\
(0.000)\end{array}$ & $\begin{array}{c}-0.0028 \\
(0.000)\end{array}$ & $\begin{array}{c}-0.0005 \\
(0.001)\end{array}$ & $\begin{array}{c}-0.0022 \\
(0.000)\end{array}$ & $\begin{array}{c}-0.0028 \\
(0.000)\end{array}$ & $\begin{array}{c}-0.0006 \\
(0.000)\end{array}$ & $\begin{array}{l}-0.0022 \\
(0.000)\end{array}$ & $\begin{array}{c}-0.0028 \\
(0.000)\end{array}$ & $\begin{array}{c}-0.0006 \\
(0.000)\end{array}$ \\
\hline Duality & $\begin{array}{l}0.0005 \\
(0.554)\end{array}$ & $\begin{array}{l}0.0005 \\
(0.681)\end{array}$ & $\begin{array}{c}-0.0000 \\
(0.997)\end{array}$ & $\begin{array}{l}0.0007 \\
(0.402)\end{array}$ & $\begin{array}{l}0.0007 \\
(0.553)\end{array}$ & $\begin{array}{l}0.0000 \\
(0.984)\end{array}$ & $\begin{array}{l}0.0005 \\
(0.570)\end{array}$ & $\begin{array}{l}0.0006 \\
(0.613)\end{array}$ & $\begin{array}{l}0.0002 \\
(0.554)\end{array}$ & $\begin{array}{l}0.0007 \\
(0.389)\end{array}$ & $\begin{array}{l}0.0007 \\
(0.515)\end{array}$ & $\begin{array}{l}0.0001 \\
(0.886)\end{array}$ \\
\hline Cash & $\begin{array}{l}0.0019 \\
(0.517)\end{array}$ & $\begin{array}{l}0.0030 \\
(0.473) \\
\end{array}$ & $\begin{array}{l}0.0011 \\
(0.527)\end{array}$ & $\begin{array}{l}0.0018 \\
(0.532)\end{array}$ & $\begin{array}{l}0.0029 \\
(0.478)\end{array}$ & $\begin{array}{l}0.0011 \\
(0.516)\end{array}$ & $\begin{array}{l}0.0015 \\
(0.620)\end{array}$ & $\begin{array}{l}0.0025 \\
(0.553)\end{array}$ & $\begin{array}{l}0.0010 \\
(0.421)\end{array}$ & $\begin{array}{l}0.0017 \\
(0.570)\end{array}$ & $\begin{array}{l}0.0029 \\
(0.479) \\
\end{array}$ & $\begin{array}{l}0.0012 \\
(0.306)\end{array}$ \\
\hline Bea & $\begin{array}{l}.0021 \\
(0.001)\end{array}$ & $\begin{array}{l}.0030 \\
(0.002)\end{array}$ & $\begin{array}{l}0.0013 \\
(0.000)\end{array}$ & $\begin{array}{l}0.0021 \\
(0.000)\end{array}$ & $\begin{array}{l}0.0030 \\
(0.001)\end{array}$ & $\begin{array}{l}.0013 \\
(0.000)\end{array}$ & $\begin{array}{l}0.0021 \\
(0.001)\end{array}$ & $\begin{array}{l}0.0029 \\
(0.001)\end{array}$ & $\begin{array}{l}.0010 \\
(0.000)\end{array}$ & $\begin{array}{l}.0021 \\
(0.000)\end{array}$ & $\begin{array}{l}.0029 \\
(0.001)\end{array}$ & $\begin{array}{l}0.0011 \\
(0.000)\end{array}$ \\
\hline Tang & $\begin{array}{l}0.0043 \\
(0.040) \\
\end{array}$ & $\begin{array}{l}0.0071 \\
(0.017) \\
\end{array}$ & $\begin{array}{l}0.0028 \\
(0.024) \\
\end{array}$ & $\begin{array}{l}0.0040 \\
(0.052) \\
\end{array}$ & $\begin{array}{l}0.0070 \\
(0.020) \\
\end{array}$ & $\begin{array}{l}0.0029 \\
(0.021) \\
\end{array}$ & $\begin{array}{l}0.0044 \\
(0.058) \\
\end{array}$ & $\begin{array}{l}0.0073 \\
(0.040) \\
\end{array}$ & $\begin{array}{l}0.0028 \\
(0.049) \\
\end{array}$ & $\begin{array}{l}0.0041 \\
(0.086) \\
\end{array}$ & $\begin{array}{l}0.0070 \\
(0.054) \\
\end{array}$ & $\begin{array}{l}0.0029 \\
(0.048) \\
\end{array}$ \\
\hline Lev & $\begin{array}{l}0.0019 \\
(0.480)\end{array}$ & $\begin{array}{l}0.0012 \\
(0.761)\end{array}$ & $\begin{array}{l}-0.0007 \\
(0.655)\end{array}$ & $\begin{array}{l}0.0024 \\
(0.361)\end{array}$ & $\begin{array}{l}0.0016 \\
(0.676)\end{array}$ & $\begin{array}{c}-0.0008 \\
(0.603)\end{array}$ & $\begin{array}{l}0.0022 \\
(0.370)\end{array}$ & $\begin{array}{l}0.0022 \\
(0.510)\end{array}$ & $\begin{array}{l}0.0004 \\
(0.694)\end{array}$ & $\begin{array}{l}0.0025 \\
(0.316)\end{array}$ & $\begin{array}{l}0.0016 \\
(0.657)\end{array}$ & $\begin{array}{c}-0.0006 \\
(0.692)\end{array}$ \\
\hline MB & $\begin{array}{c}-0.0000 \\
(0.268)\end{array}$ & $\begin{array}{l}-0.0000 \\
(0.405)\end{array}$ & $\begin{array}{c}-0.0000 \\
(0.886)\end{array}$ & $\begin{array}{c}-0.0000 \\
(0.29)\end{array}$ & $\begin{array}{c}-0.0000 \\
(0.421)\end{array}$ & $\begin{array}{c}-0.0000 \\
(0.874)\end{array}$ & $\begin{array}{c}-0.0000 \\
(0.285)\end{array}$ & $\begin{array}{l}-0.0000 \\
(0.354)\end{array}$ & $\begin{array}{c}-0.0000 \\
(0.819)\end{array}$ & $\begin{array}{c}-0.0000 \\
(0.288)\end{array}$ & $\begin{array}{c}-0.0000 \\
(0.351)\end{array}$ & $\begin{array}{c}-0.0000 \\
(0.839)\end{array}$ \\
\hline Own & $\begin{array}{c}-0.0023 \\
(0.660)\end{array}$ & $\begin{array}{c}-0.0054 \\
(0.481)\end{array}$ & $\begin{array}{c}-0.0030 \\
(0.345)\end{array}$ & $\begin{array}{c}-0.0034 \\
(0.521)\end{array}$ & $\begin{array}{c}-0.0065 \\
(0.391)\end{array}$ & $\begin{array}{c}-0.0031 \\
(0.329)\end{array}$ & $\begin{array}{c}-0.0027 \\
(0.585)\end{array}$ & $\begin{array}{c}-0.0067 \\
(0.341)\end{array}$ & $\begin{array}{c}-0.0045 \\
(0.063)\end{array}$ & $\begin{array}{c}-0.0034 \\
(0.497)\end{array}$ & $\begin{array}{c}-0.0065 \\
(0.357)\end{array}$ & $\begin{array}{c}-0.0034 \\
(0.211)\end{array}$ \\
\hline Constants & $\begin{array}{l}0.0788 \\
(0.000)\end{array}$ & $\begin{array}{l}0.0962 \\
(0.000)\end{array}$ & $\begin{array}{l}0.0174 \\
(0.019)\end{array}$ & $\begin{array}{l}.0808 \\
(0.000)\end{array}$ & $\begin{array}{l}0.1002 \\
(0.000)\end{array}$ & $\begin{array}{l}0.0195 \\
(0.008)\end{array}$ & $\begin{array}{l}0.0791 \\
(0.000)\end{array}$ & $\begin{array}{l}0.0979 \\
(0.000)\end{array}$ & $\begin{array}{l}0.0200 \\
(0.000)\end{array}$ & $\begin{array}{l}0.0807 \\
(0.000)\end{array}$ & $\begin{array}{l}0.1002 \\
(0.000)\end{array}$ & $\begin{array}{l}.0198 \\
(0.000)\end{array}$ \\
\hline Obs & 1197 & 1197 & 1197 & 1197 & 1197 & 1197 & 1197 & 1197 & 1197 & 1197 & 1197 & 1197 \\
\hline R-squared & 0.069 & 0.051 & 0.014 & 0.066 & 0.049 & 0.013 & 0.067 & 0.046 & 0.020 & 0.066 & 0.049 & 0.010 \\
\hline Sargan & 0.434 & 0.384 & 0.441 & 0.859 & 0.98 & 0.814 & & & & & & \\
\hline Endogen & 0.081 & 0.056 & 0.101 & 0.048 & 0.037 & 0.094 & & & & & & \\
\hline Hansen's J & & & & & & & 0.436 & 0.354 & 0.358 & 0.856 & 0.979 & 0.745 \\
\hline
\end{tabular}

This table presents 2SLS and one step GMM estimation on the relationship between female executive (non-executive) directors and downside risk. Sargan presents the $p$-value for the test of over-identifying restrictions with the null of the validity of the instruments. Endogen presents the $p$-values of the Wooldridge score test of endogeneity with the null hypothesis if the exogeneity of the variables. Hansen' J presents the $p$-value for the test of over-identifying restrictions with the null of the validity of the instruments. T statistics in parentheses. ${ }^{*} p<0.1$. 
Table 7. Quantile regression of females on board and risk-taking.

\begin{tabular}{|c|c|c|c|c|c|c|c|c|c|c|c|c|}
\hline & (1) & (2) & (3) & (4) & (5) & (6) & (7) & (8) & (9) & (10) & (11) & (12) \\
\hline & \multicolumn{4}{|c|}{ Individual Risk (IR) } & \multicolumn{4}{|c|}{ Systemic Risk (SR) } & \multicolumn{4}{|c|}{ Contribution Risk (CSR) } \\
\hline \multicolumn{13}{|c|}{ Panel A: High-Risk Firm (75\%) } \\
\hline Senior $_{t-1}$ & $\begin{array}{l}-0.0038 \\
(0.140) \\
\end{array}$ & & & & $\begin{array}{c}-0.0039 \\
(0.222) \\
\end{array}$ & & & & $\begin{array}{l}-0.0007 \\
(0.293) \\
\end{array}$ & & & \\
\hline Nonsenior $_{t-1}$ & & $\begin{array}{c}-0.0074^{* * *} \\
(0.000)\end{array}$ & & & & $\begin{array}{c}-0.0090^{* * *} \\
(0.001)\end{array}$ & & & & $\begin{array}{c}-0.0015^{* * *} \\
(0.002)\end{array}$ & & \\
\hline$C E O_{t-1}$ & & & $\begin{array}{c}-0.0025 * \\
(0.063)\end{array}$ & & & & $\begin{array}{c}-0.0023 \\
(0.182) \\
\end{array}$ & & & & $\begin{array}{c}-0.0003 \\
(0.271) \\
\end{array}$ & \\
\hline Chair $_{t-1}$ & & & & $\begin{array}{c}-0.0031 \text { ** } \\
(0.019)\end{array}$ & & & & $\begin{array}{c}-0.0028^{*} \\
(0.085)\end{array}$ & & & & $\begin{array}{c}-0.0006^{* *} \\
(0.037)\end{array}$ \\
\hline Manager & $\begin{array}{l}-0.0023 \\
(0.070)\end{array}$ & $\begin{array}{l}-0.0017 \\
(0.173)\end{array}$ & $\begin{array}{l}-0.0024 \\
(0.052)\end{array}$ & $\begin{array}{c}-0.0019 \\
(0.133)\end{array}$ & $\begin{array}{l}-0.0029 \\
(0.067)\end{array}$ & $\begin{array}{c}-0.0013 \\
(0.39)\end{array}$ & $\begin{array}{c}-0.0025 \\
(0.12)\end{array}$ & $\begin{array}{c}-0.0019 \\
(0.222)\end{array}$ & $\begin{array}{l}-0.0003 \\
(0.312)\end{array}$ & $\begin{array}{l}-0.0002 \\
(0.374)\end{array}$ & $\begin{array}{l}-0.0004 \\
(0.198)\end{array}$ & $\begin{array}{c}-0.0003 \\
(0.292)\end{array}$ \\
\hline Board & $\begin{array}{l}0.0004 \\
(0.777)\end{array}$ & $\begin{array}{l}0.0013 \\
(0.376)\end{array}$ & $\begin{array}{l}0.0001 \\
(0.906)\end{array}$ & $\begin{array}{l}-0.0006 \\
(0.664)\end{array}$ & $\begin{array}{l}0.0017 \\
(0.367)\end{array}$ & $\begin{array}{l}0.0015 \\
(0.386)\end{array}$ & $\begin{array}{l}0.0006 \\
(0.742)\end{array}$ & $\begin{array}{c}-0.0003 \\
(0.871) \\
\end{array}$ & $\begin{array}{l}0.0004 \\
(0.298)\end{array}$ & $\begin{array}{l}0.0003 \\
(0.264)\end{array}$ & $\begin{array}{l}0.0003 \\
(0.336)\end{array}$ & $\begin{array}{l}0.0002 \\
(0.459)\end{array}$ \\
\hline Achair & $\begin{array}{c}-0.0034 \\
(0.173)\end{array}$ & $\begin{array}{c}-0.0020 \\
(0.397)\end{array}$ & $\begin{array}{c}-0.0024 \\
(0.319)\end{array}$ & $\begin{array}{c}-0.0013 \\
(0.609)\end{array}$ & $\begin{array}{c}-0.0036 \\
(0.249) \\
\end{array}$ & $\begin{array}{c}-0.0007 \\
(0.805)\end{array}$ & $\begin{array}{c}-0.0023 \\
(0.468)\end{array}$ & $\begin{array}{c}-0.0010 \\
(0.748)\end{array}$ & $\begin{array}{l}-0.0002 \\
(0.710)\end{array}$ & $\begin{array}{l}0.0001 \\
(0.739)\end{array}$ & $\begin{array}{l}-0.0001 \\
(0.807)\end{array}$ & $\begin{array}{c}-0.0004 \\
(0.513)\end{array}$ \\
\hline Aceo & $\begin{array}{c}-0.0022 \\
(0.321)\end{array}$ & $\begin{array}{c}-0.0028 \\
(0.192) \\
\end{array}$ & $\begin{array}{c}-0.0027 \\
(0.207)\end{array}$ & $\begin{array}{c}-0.0035 \\
(0.128) \\
\end{array}$ & $\begin{array}{c}-0.0034 \\
(0.221) \\
\end{array}$ & $\begin{array}{c}-0.0031 \\
(0.259) \\
\end{array}$ & $\begin{array}{c}-0.0029 \\
(0.314)\end{array}$ & $\begin{array}{c}-0.0036 \\
(0.195) \\
\end{array}$ & $\begin{array}{l}0.0000 \\
(0.924)\end{array}$ & $\begin{array}{c}-0.0003 \\
(0.490)\end{array}$ & $\begin{array}{l}0.0001 \\
(0.843)\end{array}$ & $\begin{array}{c}-0.0000 \\
(0.911)\end{array}$ \\
\hline Size & $\begin{array}{l}-0.0025 \\
(0.000)\end{array}$ & $\begin{array}{c}-0.0026 \\
(0.000)\end{array}$ & $\begin{array}{c}-0.0023 \\
(0.000) \\
\end{array}$ & $\begin{array}{c}-0.0022 \\
(0.000)\end{array}$ & $\begin{array}{c}-0.0030 \\
(0.000)\end{array}$ & $\begin{array}{c}-0.0033 \\
(0.000) \\
\end{array}$ & $\begin{array}{c}-0.0029 \\
(0.000)\end{array}$ & $\begin{array}{c}-0.0028 \\
(0.000) \\
\end{array}$ & $\begin{array}{c}-0.0006 \\
(0.000)\end{array}$ & $\begin{array}{l}-0.0006 \\
(0.000)\end{array}$ & $\begin{array}{c}-0.0006 \\
(0.000)\end{array}$ & $\begin{array}{c}-0.0005 \\
(0.000) \\
\end{array}$ \\
\hline Duality & $\begin{array}{l}0.0006 \\
(0.490) \\
\end{array}$ & $\begin{array}{l}0.0020 \\
(0.036) \\
\end{array}$ & $\begin{array}{l}0.0004 \\
(0.649) \\
\end{array}$ & $\begin{array}{l}0.0009 \\
(0.347) \\
\end{array}$ & $\begin{array}{l}0.0014 \\
(0.236) \\
\end{array}$ & $\begin{array}{l}0.0014 \\
(0.228) \\
\end{array}$ & $\begin{array}{l}0.0011 \\
(0.357) \\
\end{array}$ & $\begin{array}{l}0.0011 \\
(0.364) \\
\end{array}$ & $\begin{array}{l}0.0001 \\
(0.637) \\
\end{array}$ & $\begin{array}{l}0.0002 \\
(0.349)\end{array}$ & $\begin{array}{c}-0.0000 \\
(0.924) \\
\end{array}$ & $\begin{array}{l}0.0001 \\
(0.545) \\
\end{array}$ \\
\hline Cash & $\begin{array}{c}-0.0019 \\
(0.598)\end{array}$ & $\begin{array}{c}-0.0005 \\
(0.871) \\
\end{array}$ & $\begin{array}{c}-0.0017 \\
(0.618)\end{array}$ & $\begin{array}{c}-0.0012 \\
(0.735)\end{array}$ & $\begin{array}{c}-0.0023 \\
(0.619)\end{array}$ & $\begin{array}{l}0.0004 \\
(0.923) \\
\end{array}$ & $\begin{array}{c}-0.0009 \\
(0.839) \\
\end{array}$ & $\begin{array}{c}-0.0005 \\
(0.911)\end{array}$ & $\begin{array}{l}0.0000 \\
(0.983) \\
\end{array}$ & $\begin{array}{l}0.0000 \\
(0.914) \\
\end{array}$ & $\begin{array}{c}-0.0001 \\
(0.859)\end{array}$ & $\begin{array}{c}-0.0001 \\
(0.896) \\
\end{array}$ \\
\hline Bea & $\begin{array}{l}0.0092 \\
(0.001) \\
\end{array}$ & $\begin{array}{l}0.0086 \\
(0.001)\end{array}$ & $\begin{array}{l}0.0091 \\
(0.001)\end{array}$ & $\begin{array}{l}0.0100 \\
(0.000)\end{array}$ & $\begin{array}{l}0.0122 \\
(0.000)\end{array}$ & $\begin{array}{l}0.0107 \\
(0.001)\end{array}$ & $\begin{array}{l}0.0125 \\
(0.000)\end{array}$ & $\begin{array}{l}0.0134 \\
(0.000) \\
\end{array}$ & $\begin{array}{l}0.0025 \\
(0.000)\end{array}$ & $\begin{array}{l}0.0021 \\
(0.001)\end{array}$ & $\begin{array}{l}0.0026 \\
(0.000)\end{array}$ & $\begin{array}{l}0.0025 \\
(0.000) \\
\end{array}$ \\
\hline Tang & $\begin{array}{l}0.0058 \\
(0.024) \\
\end{array}$ & $\begin{array}{l}0.0053 \\
(0.033) \\
\end{array}$ & $\begin{array}{l}0.0049 \\
(0.048) \\
\end{array}$ & $\begin{array}{l}0.0044 \\
(0.09) \\
\end{array}$ & $\begin{array}{l}0.0075 \\
(0.019) \\
\end{array}$ & $\begin{array}{l}0.0053 \\
(0.084) \\
\end{array}$ & $\begin{array}{l}0.0068 \\
(0.037) \\
\end{array}$ & $\begin{array}{l}0.0075 \\
(0.02) \\
\end{array}$ & $\begin{array}{l}0.0013 \\
(0.035) \\
\end{array}$ & $\begin{array}{l}0.0012 \\
(0.033) \\
\end{array}$ & $\begin{array}{l}0.0012 \\
(0.074) \\
\end{array}$ & $\begin{array}{l}0.0010 \\
(0.114) \\
\end{array}$ \\
\hline Lev & $\begin{array}{l}-0.0030 \\
(0.317)\end{array}$ & $\begin{array}{c}-0.0038 \\
(0.202)\end{array}$ & $\begin{array}{l}-0.0034 \\
(0.241)\end{array}$ & $\begin{array}{c}-0.0025 \\
(0.410)\end{array}$ & $\begin{array}{c}-0.0039 \\
(0.300)\end{array}$ & $\begin{array}{l}-0.0054 \\
(0.142)\end{array}$ & $\begin{array}{l}-0.0036 \\
(0.353)\end{array}$ & $\begin{array}{c}-0.0043 \\
(0.252) \\
\end{array}$ & $\begin{array}{l}-0.0006 \\
(0.426)\end{array}$ & $\begin{array}{l}-0.0008 \\
(0.216)\end{array}$ & $\begin{array}{c}-0.0003 \\
(0.676)\end{array}$ & $\begin{array}{l}-0.0006 \\
(0.373)\end{array}$ \\
\hline MB & $\begin{array}{l}0.0000 \\
(0.739)\end{array}$ & $\begin{array}{l}0.0000 \\
(0.516)\end{array}$ & $\begin{array}{l}0.0000 \\
(0.619)\end{array}$ & $\begin{array}{l}0.0000 \\
(0.452)\end{array}$ & $\begin{array}{l}0.0000 \\
(0.363)\end{array}$ & $\begin{array}{l}0.0000 \\
(0.804)\end{array}$ & $\begin{array}{l}0.0000 \\
(0.374)\end{array}$ & $\begin{array}{l}0.0000 \\
(0.325)\end{array}$ & $\begin{array}{l}0.0000 \\
(0.656)\end{array}$ & $\begin{array}{l}0.0000 \\
(0.964)\end{array}$ & $\begin{array}{l}0.0000 \\
(0.523)\end{array}$ & $\begin{array}{l}0.0000 \\
(0.436)\end{array}$ \\
\hline
\end{tabular}


Table 7. Cont

\begin{tabular}{|c|c|c|c|c|c|c|c|c|c|c|c|c|}
\hline & (1) & (2) & (3) & (4) & (5) & (6) & (7) & (8) & (9) & (10) & (11) & (12) \\
\hline & \multicolumn{4}{|c|}{ Individual Risk (IR) } & \multicolumn{4}{|c|}{ Systemic Risk (SR) } & \multicolumn{4}{|c|}{ Contribution Risk (CSR) } \\
\hline Own & $\begin{array}{l}0.0089 \\
(0.000) \\
\end{array}$ & $\begin{array}{l}0.0095 \\
(0.000) \\
\end{array}$ & $\begin{array}{l}0.0081 \\
(0.000) \\
\end{array}$ & $\begin{array}{l}0.0077 \\
(0.001) \\
\end{array}$ & $\begin{array}{l}0.0099 \\
(0.001) \\
\end{array}$ & $\begin{array}{l}0.0121 \\
(0.000) \\
\end{array}$ & $\begin{array}{l}0.0091 \\
(0.002) \\
\end{array}$ & $\begin{array}{l}0.0089 \\
(0.002) \\
\end{array}$ & $\begin{array}{l}0.0015 \\
(0.008) \\
\end{array}$ & $\begin{array}{l}0.0018 \\
(0.001)\end{array}$ & $\begin{array}{l}0.0013 \\
(0.021)\end{array}$ & $\begin{array}{l}0.0014 \\
(0.012)\end{array}$ \\
\hline Cons & $\begin{array}{l}0.1033 \\
(0.000) \\
\end{array}$ & $\begin{array}{l}0.1014 \\
(0.000) \\
\end{array}$ & $\begin{array}{l}0.0996 \\
(0.000) \\
\end{array}$ & $\begin{array}{l}0.0949 \\
(0.000) \\
\end{array}$ & $\begin{array}{l}0.1236 \\
(0.000) \\
\end{array}$ & $\begin{array}{l}0.1146 \\
(0.000) \\
\end{array}$ & $\begin{array}{l}0.1154 \\
(0.000) \\
\end{array}$ & $\begin{array}{l}0.1117 \\
(0.000) \\
\end{array}$ & $\begin{array}{l}0.0180 \\
(0.000) \\
\end{array}$ & $\begin{array}{l}0.0193 \\
(0.000) \\
\end{array}$ & $\begin{array}{l}0.0181 \\
(0.000) \\
\end{array}$ & $\begin{array}{l}0.0184 \\
(0.000) \\
\end{array}$ \\
\hline Obs & 1197 & 1197 & 1197 & 1197 & 1197 & 1197 & 1197 & 1197 & 1197 & 1197 & 1197 & 1197 \\
\hline$p-\mathrm{R} 2$ & 0.0897 & 0.0968 & 0.0918 & 0.0919 & 0.0833 & 0.0896 & 0.0847 & 0.0853 & 0.0592 & 0.0645 & 0.0599 & 0.0604 \\
\hline \multicolumn{13}{|c|}{ Panel B: Low-Risk Firm (25\%) } \\
\hline Senior $_{t-1}$ & $\begin{array}{c}-0.0059^{* * *} \\
(0.001)\end{array}$ & & & & $\begin{array}{c}-0.0070^{* * *} \\
(0.001)\end{array}$ & & & & $\begin{array}{c}-0.0009 \text { ** } \\
(0.04)\end{array}$ & & & \\
\hline Nonsenior $_{t-1}$ & & $\begin{array}{c}-0.0042^{* * *} \\
(0.005)\end{array}$ & & & & $\begin{array}{c}-0.0051^{* * *} \\
(0.007)\end{array}$ & & & & $\begin{array}{c}-0.0006^{*} \\
(0.092)\end{array}$ & & \\
\hline$C E O_{t-1}$ & & & $\begin{array}{c}-0.0011 \\
(0.235)\end{array}$ & & & & $\begin{array}{c}-0.0010 \\
(0.380)\end{array}$ & & & & $\begin{array}{c}-0.0002 \\
(0.380)\end{array}$ & \\
\hline Chair $_{t-1}$ & & & & $\begin{array}{c}-0.0023^{* * *} \\
(0.007) \\
\end{array}$ & & & & $\begin{array}{c}-0.0022 \text { ** } \\
(0.034) \\
\end{array}$ & & & & $\begin{array}{c}-0.0004 \text { * } \\
(0.057)\end{array}$ \\
\hline Manager & $\begin{array}{l}-0.0001 \\
(0.864)\end{array}$ & $\begin{array}{l}0.0005 \\
(0.544)\end{array}$ & $\begin{array}{l}0.0004 \\
(0.606)\end{array}$ & $\begin{array}{l}0.0001 \\
(0.848) \\
\end{array}$ & $\begin{array}{c}-0.0000 \\
(0.966)\end{array}$ & $\begin{array}{l}0.0005 \\
(0.601)\end{array}$ & $\begin{array}{c}-0.0000 \\
(0.99)\end{array}$ & $\begin{array}{c}-0.0005 \\
(0.62)\end{array}$ & $\begin{array}{l}-0.0001 \\
(0.447)\end{array}$ & $\begin{array}{l}-0.0001 \\
(0.637)\end{array}$ & $\begin{array}{l}-0.0001 \\
(0.506)\end{array}$ & $\begin{array}{c}-0.0001 \\
(0.478)\end{array}$ \\
\hline Board & $\begin{array}{l}0.0001 \\
(0.875)\end{array}$ & $\begin{array}{c}-0.0007 \\
(0.488)\end{array}$ & $\begin{array}{l}-0.0004 \\
(0.655)\end{array}$ & $\begin{array}{c}-0.0000 \\
(0.971)\end{array}$ & $\begin{array}{c}-0.0003 \\
(0.763)\end{array}$ & $\begin{array}{c}-0.0006 \\
(0.631)\end{array}$ & $\begin{array}{c}-0.0002 \\
(0.822)\end{array}$ & $\begin{array}{l}0.0004 \\
(0.709)\end{array}$ & $\begin{array}{l}0.0000 \\
(0.861)\end{array}$ & $\begin{array}{l}0.0000 \\
(0.771)\end{array}$ & $\begin{array}{c}-0.0000 \\
(0.794)\end{array}$ & $\begin{array}{c}-0.0000 \\
(0.953)\end{array}$ \\
\hline Achair & $\begin{array}{l}0.0003 \\
(0.854)\end{array}$ & $\begin{array}{l}0.0008 \\
(0.633)\end{array}$ & $\begin{array}{l}0.0015 \\
(0.375)\end{array}$ & $\begin{array}{l}0.0016 \\
(0.343)\end{array}$ & $\begin{array}{c}-0.0008 \\
(0.679)\end{array}$ & $\begin{array}{l}0.0011 \\
(0.615)\end{array}$ & $\begin{array}{l}0.0015 \\
(0.464)\end{array}$ & $\begin{array}{l}0.0017 \\
(0.391)\end{array}$ & $\begin{array}{l}0.0002 \\
(0.631)\end{array}$ & $\begin{array}{l}0.0001 \\
(0.753)\end{array}$ & $\begin{array}{l}0.0003 \\
(0.424)\end{array}$ & $\begin{array}{l}0.0002 \\
(0.540)\end{array}$ \\
\hline Aceo & $\begin{array}{c}-0.0022 \\
(0.142)\end{array}$ & $\begin{array}{c}-0.0019 \\
(0.212)\end{array}$ & $\begin{array}{c}-0.0022 \\
(0.158)\end{array}$ & $\begin{array}{c}-0.0023 \\
(0.121)\end{array}$ & $\begin{array}{c}-0.0026 \\
(0.139)\end{array}$ & $\begin{array}{c}-0.0024 \\
(0.223)\end{array}$ & $\begin{array}{c}-0.0027 \\
(0.161)\end{array}$ & $\begin{array}{c}-0.0022 \\
(0.214)\end{array}$ & $\begin{array}{c}-0.0001 \\
(0.758)\end{array}$ & $\begin{array}{c}-0.0000 \\
(0.889)\end{array}$ & $\begin{array}{c}-0.0000 \\
(0.844)\end{array}$ & $\begin{array}{c}-0.0000 \\
(0.807)\end{array}$ \\
\hline Size & $\begin{array}{c}-0.0013 \\
(0.000)\end{array}$ & $\begin{array}{c}-0.0013 \\
(0.000)\end{array}$ & $\begin{array}{c}-0.0014 \\
(0.000)\end{array}$ & $\begin{array}{c}-0.0014 \\
(0.000)\end{array}$ & $\begin{array}{c}-0.0017 \\
(0.000)\end{array}$ & $\begin{array}{c}-0.0017 \\
(0.000)\end{array}$ & $\begin{array}{c}-0.0017 \\
(0.000)\end{array}$ & $\begin{array}{c}-0.0017 \\
(0.000)\end{array}$ & $\begin{array}{c}-0.0003 \\
(0.000)\end{array}$ & $\begin{array}{c}-0.0003 \\
(0.000)\end{array}$ & $\begin{array}{c}-0.0003 \\
(0.000)\end{array}$ & $\begin{array}{c}-0.0003 \\
(0.000) \\
\end{array}$ \\
\hline Duality & $\begin{array}{c}-0.0000 \\
(0.989)\end{array}$ & $\begin{array}{c}-0.0002 \\
(0.737)\end{array}$ & $\begin{array}{c}-0.0004 \\
(0.519)\end{array}$ & $\begin{array}{c}-0.0002 \\
(0.759)\end{array}$ & $\begin{array}{c}-0.0004 \\
(0.601)\end{array}$ & $\begin{array}{c}-0.0001 \\
(0.889)\end{array}$ & $\begin{array}{c}-0.0001 \\
(0.849)\end{array}$ & $\begin{array}{c}-0.0000 \\
(0.969)\end{array}$ & $\begin{array}{c}-0.0000 \\
(0.834) \\
\end{array}$ & $\begin{array}{l}0.0000 \\
(0.942) \\
\end{array}$ & $\begin{array}{c}-0.0000 \\
(0.816) \\
\end{array}$ & $\begin{array}{c}-0.0000 \\
(0.963) \\
\end{array}$ \\
\hline Cash & $\begin{array}{l}0.0007 \\
(0.759)\end{array}$ & $\begin{array}{l}0.0002 \\
(0.921)\end{array}$ & $\begin{array}{l}0.0014 \\
(0.559)\end{array}$ & $\begin{array}{l}0.0004 \\
(0.848)\end{array}$ & $\begin{array}{l}0.0020 \\
(0.482)\end{array}$ & $\begin{array}{l}0.0025 \\
(0.43)\end{array}$ & $\begin{array}{l}0.0042 \\
(0.185)\end{array}$ & $\begin{array}{l}0.0029 \\
(0.326)\end{array}$ & $\begin{array}{l}0.0010 \\
(0.085)\end{array}$ & $\begin{array}{l}0.0009 \\
(0.147)\end{array}$ & $\begin{array}{l}0.0007 \\
(0.225)\end{array}$ & $\begin{array}{l}0.0008 \\
(0.158)\end{array}$ \\
\hline Bea & $\begin{array}{l}0.0036 \\
(0.045) \\
\end{array}$ & $\begin{array}{l}0.0033 \\
(0.075) \\
\end{array}$ & $\begin{array}{l}0.0028 \\
(0.132) \\
\end{array}$ & $\begin{array}{l}0.0035 \\
(0.055) \\
\end{array}$ & $\begin{array}{c}0.0049 \\
(0.02)\end{array}$ & $\begin{array}{l}0.0040 \\
(0.09) \\
\end{array}$ & $\begin{array}{l}0.0038 \\
(0.096) \\
\end{array}$ & $\begin{array}{l}0.0047 \\
(0.033) \\
\end{array}$ & $\begin{array}{l}0.0007 \\
(0.085) \\
\end{array}$ & $\begin{array}{l}0.0009 \\
(0.059) \\
\end{array}$ & $\begin{array}{l}0.0006 \\
(0.124) \\
\end{array}$ & $\begin{array}{l}0.0010 \\
(0.027) \\
\end{array}$ \\
\hline Tang & $\begin{array}{l}0.0016 \\
(0.348)\end{array}$ & $\begin{array}{l}0.0016 \\
(0.351)\end{array}$ & $\begin{array}{l}0.0009 \\
(0.581)\end{array}$ & $\begin{array}{l}0.0005 \\
(0.755)\end{array}$ & $\begin{array}{l}0.0022 \\
(0.252)\end{array}$ & $\begin{array}{l}0.0020 \\
(0.367)\end{array}$ & $\begin{array}{l}0.0022 \\
(0.307)\end{array}$ & $\begin{array}{l}0.0022 \\
(0.275)\end{array}$ & $\begin{array}{l}0.0004 \\
(0.297)\end{array}$ & $\begin{array}{l}0.0005 \\
(0.257)\end{array}$ & $\begin{array}{l}0.0004 \\
(0.303)\end{array}$ & $\begin{array}{l}0.0005 \\
(0.229)\end{array}$ \\
\hline
\end{tabular}


Table 7. Cont.

\begin{tabular}{|c|c|c|c|c|c|c|c|c|c|c|c|c|}
\hline & (1) & (2) & (3) & (4) & (5) & (6) & (7) & (8) & (9) & (10) & (11) & (12) \\
\hline & \multicolumn{4}{|c|}{ Individual Risk (IR) } & \multicolumn{4}{|c|}{ Systemic Risk (SR) } & \multicolumn{4}{|c|}{ Contribution Risk (CSR) } \\
\hline Lev & $\begin{array}{l}0.0010 \\
(0.603)\end{array}$ & $\begin{array}{l}0.0010 \\
(0.631)\end{array}$ & $\begin{array}{l}0.0018 \\
(0.368)\end{array}$ & $\begin{array}{l}0.0029 \\
(0.150)\end{array}$ & $\begin{array}{l}0.0009 \\
(0.682)\end{array}$ & $\begin{array}{l}0.0019 \\
(0.475)\end{array}$ & $\begin{array}{l}0.0020 \\
(0.433)\end{array}$ & $\begin{array}{l}0.0030 \\
(0.222)\end{array}$ & $\begin{array}{l}0.0000 \\
(0.907)\end{array}$ & $\begin{array}{c}-0.0002 \\
(0.582)\end{array}$ & $\begin{array}{c}-0.0000 \\
(0.932)\end{array}$ & $\begin{array}{c}-0.0000 \\
(0.933)\end{array}$ \\
\hline MB & $\begin{array}{c}-0.0001 \\
(0.005) \\
\end{array}$ & $\begin{array}{c}-0.0001 \\
(0.006) \\
\end{array}$ & $\begin{array}{c}-0.0001 \\
(0.002) \\
\end{array}$ & $\begin{array}{c}-0.0000 \\
(0.013) \\
\end{array}$ & $\begin{array}{c}-0.0001 \\
(0.006) \\
\end{array}$ & $\begin{array}{c}-0.0001 \\
(0.011) \\
\end{array}$ & $\begin{array}{c}-0.0001 \\
(0.006) \\
\end{array}$ & $\begin{array}{c}-0.0001 \\
(0.009) \\
\end{array}$ & $\begin{array}{c}-0.0000 \\
(0.036) \\
\end{array}$ & $\begin{array}{c}-0.0000 \\
(0.012) \\
\end{array}$ & $\begin{array}{c}-0.0000 \\
(0.014) \\
\end{array}$ & $\begin{array}{c}-0.0000 \\
(0.042) \\
\end{array}$ \\
\hline Own & $\begin{array}{l}0.0022 \\
(0.141) \\
\end{array}$ & $\begin{array}{l}0.0025 \\
(0.106) \\
\end{array}$ & $\begin{array}{l}0.0025 \\
(0.106) \\
\end{array}$ & $\begin{array}{l}0.0024 \\
(0.114) \\
\end{array}$ & $\begin{array}{l}0.0008 \\
(0.618) \\
\end{array}$ & $\begin{array}{l}0.0018 \\
(0.364) \\
\end{array}$ & $\begin{array}{c}0.0015 \\
(0.44) \\
\end{array}$ & $\begin{array}{l}0.0012 \\
(0.514) \\
\end{array}$ & $\begin{array}{c}-0.0002 \\
(0.544) \\
\end{array}$ & $\begin{array}{c}-0.0001 \\
(0.749)\end{array}$ & $\begin{array}{c}-0.0001 \\
(0.652)\end{array}$ & $\begin{array}{c}-0.0002 \\
(0.562)\end{array}$ \\
\hline Cons & $\begin{array}{l}0.0562 \\
(0.000)\end{array}$ & $\begin{array}{l}0.0541 \\
(0.000)\end{array}$ & $\begin{array}{l}0.0534 \\
(0.000)\end{array}$ & $\begin{array}{l}0.0524 \\
(0.000)\end{array}$ & $\begin{array}{l}0.0757 \\
(0.000)\end{array}$ & $\begin{array}{l}0.0675 \\
(0.000)\end{array}$ & $\begin{array}{l}0.0654 \\
(0.000)\end{array}$ & $\begin{array}{l}0.0629 \\
(0.000)\end{array}$ & $\begin{array}{l}0.0120 \\
(0.000)\end{array}$ & $\begin{array}{l}0.0117 \\
(0.000)\end{array}$ & $\begin{array}{l}0.0109 \\
(0.000)\end{array}$ & $\begin{array}{l}0.0112 \\
(0.000)\end{array}$ \\
\hline Obs & 1197 & 1197 & 1197 & 1197 & 1197 & 1197 & 1197 & 1197 & 1197 & 1197 & 1197 & 1197 \\
\hline$p-\mathrm{R} 2$ & 0.0567 & 0.0577 & 0.0531 & 0.056 & 0.053 & 0.0542 & 0.0499 & 0.0523 & 0.0452 & 0.0443 & 0.0427 & 0.0455 \\
\hline
\end{tabular}

This table presents ordinary least squares (OLS) quantile regressions with bootstrapped standard errors. Q75 at the 75th percentile and Q25 at the 25th percentile. All regressions include dummies for a year. ${ }^{*} p<0.1$; ${ }^{* *} p<0.05$; ${ }^{* *} p<0.01$. $p$-R2 denotes pseudo R-squared. 


\section{Discussion and Implications}

The occurrence of the financial crisis has made risk management become the important element in business [24]. It is clearly mentioned that good corporate governance alleviates the downside risk of firms and even financial market $[4,6]$. By considering women in alternative managerial levels incorporate risk-taking, this study aims to consolidate and extend the results of [1]. We consider compositing of three risk attributes (i.e., individual risk, systemic risk, and contribution risk) which are measured by parametric-based value at risk (VaR) and expected shortfall.

To answer the research question on the role of corporate governance in risk management, especially in Vietnam as a frontier market, our main argument is that women could reduce the agency problem in firm management, which enhances their impact on executive decision-making even in reducing downside risk [12,56]. Similarly, ref. [84] showed that female managers improve effective collaboration among all board members that lead to an increase in accounting return [85]. In such a context of gender diversity and downside risk, our empirical results indicate that the risk attributes reduction is mainly driven by female non-senior managers who provide more specific expertise with their conservative characteristics for board's decisions as they are appointed [65]. Besides, we provide the evidence for the female CEO and chairman to increase the IR and SR It explains that the female top executives do the business orientation in manufacturing firms with fewer risk strategies. Therefore, their experience and education will influence the board in identifying effective risk-considering actions [86]. In addition, we also found that women are limited their involvement to a firm's strategy if they are appointed as non-executive directors [32]. Otherwise, the female executive director has a negative impact on firm risk (IR). In this process, the stand IR is important because the risk is the main component to achieve a successful investment strategy that defines the firm's industrial competitiveness [14]. On the other hand, the SR and CSR were marked as the critical factors that lead to the financial crisis [5]. The relationship between gender diversity and downside risk is also determined whether firms are high or low risk-takers. We find that women have more impact on reducing excessive risk-taking at both senior and non-senior management in low-risk firms, but it does not support the case of female CEO. This is probably due to the fact that they are less prone to engage in activities as CEO with uncertain economic results or against the desires of the firm.

Consequently, our study successfully links gender diversity with the firm's risk management, especially downside risk. We apply the comprehensive proxy measure of gender diversity that involves female managers and executives. Our results demonstrate that a high proportion of women in alternative firm managerial levels lowers risk attributes, which are proxies for downside risk. In a context such as developing Asian countries, the proportion of women is slightly lower firm management level, our study is very important, once it shows the vital role of women in reducing the firm's downside risk [3]. Therefore, the policymakers, specifically in countries that are similar to Vietnam, should control the proportion of women in firm managerial levels to ensure gender equality and systematic risks. However, this study does not mention the quotas to achieve gender equality in the management structure. On the other hand, the appearance of women may not relevant to a firm's value-enhancing strategy [81]. In such a case, women tend to take less risk strategy rather than maximize managerial incentives from increase firm risk. Therefore, high-risk firms may consider increasing the number of women in management to lower the risk.

From this study, we provide the most important findings on the role of gender diversity, not only onboard but also on firm management in reducing downside risk. As with most research, we mention several limitations for future studies. First, we restrict the data from only Vietnamese listed companies which represent for frontier markets. Future studies may include different industrial sectors or countries. Second, we restrict women only on executive and management levels. Future work may consider clarifying their role in alternative positions (e.g., institutional ownership).

Author Contributions: Supervision, Y.-H.W.; Writing-original draft, C.-D.T. and M.-T.P.; Writing一review \& editing, F.-J.Y. All authors have read and agreed to the published version of the manuscript. 
Funding: This research received no external funding.

Acknowledgments: Thanks to the suggestions and comments of three anonymous reviewers, whose great job improves the quality of this paper. However, all the faults and deficiencies belong to the authors.

Conflicts of Interest: The authors declare no conflict of interest.

\section{References}

1. Wang, J.-Y.; Tran, C.-D.; Wu, W.-L. Role of Board Structure in Financial Institutions: Evidence from Western Europe. J. Account. Financ. Manag. Strategy 2019, 14, 113-151.

2. Ionascu, M.; Ionascu, I.; Sacarin, M.; Minu, M. Women on Boards and Financial Performance: Evidence from a European Emerging Market. Sustainability 2018, 10, 1644. [CrossRef]

3. Thornton, G. Women in Business: Building a Blueprint for Action; Grant Thornton Grant Thornton International Ltd.: London, UK, 2019.

4. Andries, A.M.; Nistor, S. Systemic risk, corporate governance and regulation of banks across emerging countries. Econ. Lett. 2016, 144, 59-63. [CrossRef]

5. IMF. Sudan: 2006 Article IV Consultation and Staff-Monitored Program-Staff Report; Staff Statement; Public Information Notice on the Executive Board Discussion; and Statement by the Executive Director for Sudan; International Monetary Fund: Washington, DC, USA, 2014.

6. Wang, L.-H.; Lin, C.-H.; Fung, H.-G.; Chen, H.-M. Governance mechanisms and downside risk. Pac. Basin Financ. J. 2015, 35, 485-498. [CrossRef]

7. Jensen, M.C.; Smith, J.C.W. Stockholder, Manager, and Creditor Interests: Applications of Agency Theory. SSRN Electron. J. 2000, 1. [CrossRef]

8. Kirkpatrick, G. The corporate governance lessons from the financial crisis. OECD J. Financ. Mark. Trends 2009, 2009, 61-87. [CrossRef]

9. Cheng, S. Board size and the variability of corporate performance. J. Financ. Econ. 2008, 87, 157-176. [CrossRef]

10. Erkens, D.H.; Hung, M.Y.; Matos, P. Corporate governance in the 2007-2008 financial crisis: Evidence from financial institutions worldwide. J. Corp. Financ. 2012, 18, 389-411. [CrossRef]

11. Huang, J.; Kisgen, D.J. Gender and corporate finance: Are male executives overconfident relative to female executives? J. Financ. Econ. 2013, 108, 822-839. [CrossRef]

12. Berger, A.N.; Kick, T.; Schaeck, K. Executive board composition and bank risk taking. J. Corp. Financ. 2014, 28, 48-65. [CrossRef]

13. Loukil, N.; Yousfi, O. Does gender diversity on corporate boards increase risk-taking? Can. J. Adm. Sci. Rev. Can. Sci. Adm. 2015, 33, 66-81. [CrossRef]

14. Sila, V.; Gonzalez, A.; Hagendorff, J. Women on board: Does boardroom gender diversity affect firm risk? J. Corp. Financ. 2016, 36, 26-53. [CrossRef]

15. Wiseman, R.M.; Gomez-Mejia, L.R. A behavioral agency model of managerial risk taking. Acad. Manag. Rev. 1998, 23, 133-153. [CrossRef]

16. Boubaker, S.; Nguyen, P.; Rouatbi, W. Multiple Large Shareholders and Corporate Risk-taking: Evidence from French Family Firms. Eur. Financ. Manag. 2016, 22, 697-745. [CrossRef]

17. Jorion, P. The New Benchmark for Managing Financial Risk: Value at Risk; McGraw-Hill: New York, NY, USA, 2001.

18. Krause, A. Coherent risk measurement: An introduction. Balance Sheet 2002, 10, 13-17. [CrossRef]

19. Liao, Y. The benefit of modeling jumps in realized volatility for risk prediction: Evidence from Chinese mainland stocks. Pac. Basin Financ. J. 2013, 23, 25-48. [CrossRef]

20. Inui, K.; Kijima, M. On the significance of expected shortfall as a coherent risk measure. J. Bank. Financ. 2005, 29, 853-864. [CrossRef]

21. Engle, R. Risk and Volatility: Econometric Models and Financial Practice. Am. Econ. Rev. 2004, 94, 405-420. [CrossRef]

22. Cai, Z.; Wang, X. Nonparametric estimation of conditional VaR and expected shortfall. J. Econ. 2008, 147, 120-130. [CrossRef]

23. Fan, J.; Gu, J. Semiparametric estimation of Value at Risk. Econ. J. 2003, 6, 261-290. [CrossRef]

24. Pfaff, B. Financial Risk Modelling and Portfolio Optimization with R; Wiley: Hoboken, NJ, USA, 2016. 
25. Luethi, D.; Breymann, W. Ghyp: A Package on Generalized Hyperbolic Distribution and Its Special Cases. 2016. Available online: https://rdrrio/rforge/ghyp/ (accessed on 10 April 2020).

26. Poletti-Hughes, J.; Turrent, G.D.C.B. Gender diversity on the board of directors and corporate risk: A behavioural agency theory perspective. Int. Rev. Financ. Anal. 2019, 62, 80-90. [CrossRef]

27. Wright, P.; Ferris, S.P.; Sarin, A.; Awasthi, V. Impact of corporate insider, blockholder, and institutional equity ownership on firm risk taking. Acad. Manag. J. 1996, 39, 441-458.

28. Anderson, C.W.; Campbell, T.L. Corporate governance of Japanese banks. J. Corp. Financ. 2004, 10, 327-354. [CrossRef]

29. Laeven, L.; Levine, R. Bank governance, regulation and risk taking. J. Financ. Econ. 2009, 93, $259-275$. [CrossRef]

30. Gadhoum, Y.; Ayadi, M.A. Ownership structure and risk: A Canadian empirical analysis. Q. J. Bus. Econ. 2003, 42, 19-39.

31. Tran, N.H.; Le, C.D. Ownership concentration, corporate risk-taking and performance: Evidence from Vietnamese listed firms. Cogent Econ. Financ. 2020, 8, 1732640. [CrossRef]

32. A Wearing, C.; Wearing, B. Between glass ceilings: Female non-executive directors in UK quoted companies. Int. J. Discl. Gov. 2004, 1, 355-371. [CrossRef]

33. Cruz, C.; Justo, R.; Larraza-Kintana, M.; Garcés-Galdeano, L. When Do Women Make a Better Table? Examining the Influence of Women Directors on Family Firm's Corporate Social Performance. Entrep. Theory Pr. 2018, 43, 282-301. [CrossRef]

34. Nahum, N.; Carmeli, A. Leadership style in a board of directors: Implications of involvement in the strategic decision-making process. J. Manag. Gov. 2019, 24, 199-227. [CrossRef]

35. Young, M.N.; Peng, M.W.; Ahlstrom, D.; Bruton, G.D.; Jiang, Y. Corporate Governance in Emerging Economies: A Review of the Principal-Principal Perspective. J. Manag. Stud. 2008, 45, 196-220. [CrossRef]

36. Vo, D.H.; Nguyen, T.M. The Impact of Corporate Governance on Firm Performance: Empirical Study in Vietnam. Int. J. Econ. Financ. 2014, 6, 1-13. [CrossRef]

37. Nguyen, T.; Locke, S.; Reddy, K. Does boardroom gender diversity matter? Evidence from a transitional economy. Int. Rev. Econ. Financ. 2015, 37, 184-202. [CrossRef]

38. Munro, J. Women's Representation in Leadership in Viet Nam; United Nations Development Programme: New York, NY, USA, 2012.

39. Fama, E.F. Agency Problems and the Theory of the Firm. J. Political Econ. 1980, 88, 288-307. [CrossRef]

40. Jensen, M.C.; Meckling, W. Theory of the firm: Managerial behavior, agency costs and ownership structure. J. Financ. Econ. 1976, 3, 305-360. [CrossRef]

41. Baysinger, B.; Hoskisson, R.E. The composition of boards of directors and strategic control: Effects on corporate strategy. Acad. Manag. Rev. 1990, 15, 72-87. [CrossRef]

42. Jensen, M.C.; Murphy, K.J. Performance Pay and Top-Management Incentives. J. Political Econ. 1990, 98, 225-264. [CrossRef]

43. Pathan, S. Strong boards, CEO power and bank risk-taking. J. Bank. Financ. 2009, 33, 1340-1350. [CrossRef]

44. Nam, S.-W.; Nam, I.C. Corporate Governance in Asia; Asian Development Bank Institute: Tokyo, Japan, 2004; pp. 1-211.

45. Staikouras, P.K.; Staikouras, C.K.; Agoraki, M.-E.K. The effect of board size and composition on European bank performance. Eur. J. Law Econ. 2007, 23, 1-27. [CrossRef]

46. Levine, R. The Corporate Governance of Banks: A Concise Discussion of Concepts and Evidence; The World Bank: Washington, DC, USA, 2004.

47. Prowse, S. Corporate control in commercial banks. J. Financ. Res. 1997, 20, 509-527. [CrossRef]

48. Adams, R.B.; E Hermalin, B.; Weisbach, M.S. The Role of Boards of Directors in Corporate Governance: A Conceptual Framework and Survey. J. Econ. Lit. 2010, 48, 58-107. [CrossRef]

49. Saeed, A.; Yousaf, A.; Alharbi, J. Family and state ownership, internationalization and corporate board-gender diversity. Cross Cult. Strat. Manag. 2017, 24, 251-270. [CrossRef]

50. Galbreath, J. Are there gender-related influences on corporate sustainability? A study of women on boards of directors. J. Manag. Organ. 2011, 17, 17-38. [CrossRef]

51. Sunden, A.E.; Surette, B.J. Gender differences in the allocation of assets in retirement savings plans. Am. Econ. Rev. 1998, 88, 207-211.

52. Croson, R.; Gneezy, U. Gender Differences in Preferences. J. Econ. Lit. 2009, 47, 448-474. [CrossRef] 
53. Ertac, S.; Gurdal, M.Y. Deciding to decide: Gender, leadership and risk-taking in groups. J. Econ. Behav. Organ. 2012, 83, 24-30. [CrossRef]

54. Olsen, R.A.; Cox, C.M. The Influence of Gender on the Perception and Response to Investment Risk: The Case of Professional Investors. J. Psychol. Financ. Mark. 2001, 2, 29-36. [CrossRef]

55. Bandiera, O.; Guiso, L.; Prat, A.; Sadun, R. Matching Firms, Managers, and Incentives. J. Labor Econ. 2015, 33, 623-681. [CrossRef]

56. Perryman, A.A.; Fernando, G.D.; Tripathy, A. Do gender differences persist? An examination of gender diversity on firm performance, risk, and executive compensation. J. Bus. Res. 2016, 69, 579-586. [CrossRef]

57. Carli, L.L.; Eagly, A.H. Women face a labyrinth: An examination of metaphors for women leaders. Gend. Manag. Int. J. 2016, 31, 514-527. [CrossRef]

58. Francoeur, C.; Labelle, R.; Sinclair-Desgagné, B. Gender Diversity in Corporate Governance and Top Management. J. Bus. Ethics 2007, 81, 83-95. [CrossRef]

59. Bennouri, M.; Chtioui, T.; Nagati, H.; Nekhili, M. Female board directorship and firm performance: What really matters? J. Bank. Financ. 2018, 88, 267-291. [CrossRef]

60. Carter, D.A.; Simkins, B.J.; Simpson, W.G. Corporate Governance, Board Diversity, and Firm Value. Financ. Rev. 2003, 38, 33-53. [CrossRef]

61. Eagly, A.H.; Carli, L.L. The female leadership advantage: An evaluation of the evidence. Leadersh. Q. 2003, 14, 807-834. [CrossRef]

62. Bonn, I.; Yoshikawa, T.; Phan, P.H. Effects of Board Structure on Firm Performance: A Comparison between Japan and Australia. Asian Bus. Manag. 2004, 3, 105-125. [CrossRef]

63. Mukarram, S.S.; Saeed, A.; Hammoudeh, S.; Raziq, M.M. Women on Indian boards and market performance: A role-congruity theory perspective. Asian Bus. Manag. 2018, 17, 4-36. [CrossRef]

64. Eagly, A.H.; Johnson, B.T. Gender and leadership style: A meta-analysis. Psychol. Bull. 1990, 108, 233. [CrossRef]

65. Fondas, N.; Sassalos, S. A different voice in the boardroom: How the presence of women directors affects board influence over management. Glob. Focus 2000, 12, 13-22.

66. Hillman, A.J.; Cannella, A.A., Jr.; Harris, I.C. Women and racial minorities in the boardroom: How do directors differ? J. Manag. 2002, 28,747-763. [CrossRef]

67. Melero, E. Are workplaces with many women in management run differently? J. Bus. Res. 2011, 64, $385-393$. [CrossRef]

68. Martin, A.D.; Nishikawa, T.; Williams, M.A. CEO gender: Effects on valuation and risk. Q. J. Financ. Account. 2009, 48, 23-40.

69. Faccio, M.; Marchica, M.; Mura, R. CEO gender, corporate risk-taking, and the efficiency of capital allocation. J. Corp. Financ. 2016, 39, 193-209. [CrossRef]

70. Abbott, P.; Tarp, F. Globalization crises, trade and development in Vietnam. J. Int. Commer. Econ. Policy 2012, 3, 1240006. [CrossRef]

71. Riedel, J. The Global Economic Crisis and Its Long-Run Implications for Vietnam; United Nations Development Programme: New York, NY, USA, 2009.

72. Alexander, G.J.; Baptista, A. Economic implications of using a mean-VaR model for portfolio selection: A comparison with mean-variance analysis. J. Econ. Dyn. Control. 2002, 26, 1159-1193. [CrossRef]

73. Baumol, W.J. An Expected Gain-Confidence Limit Criterion for Portfolio Selection. Manag. Sci. 1963, 10, 174-182. [CrossRef]

74. Campbell, K.; Vera, A.M. Female board appointments and firm valuation: Short and long-term effects. J. Manag. Gov. 2009, 14, 37-59. [CrossRef]

75. Wintoki, M.B.; Linck, J.S.; Netter, J.M. Endogeneity and the dynamics of internal corporate governance. J. Financ. Econ. 2012, 105, 581-606. [CrossRef]

76. Kupiec, P.H. Techniques for Verifying the Accuracy of Risk Measurement Models. J. Deriv. 1995, 3, 73-84. [CrossRef]

77. Campbell, J.Y.; Hilscher, J.; Szilagyi, J. In Search of Distress Risk. J. Financ. 2008, 63, 2899-2939. [CrossRef]

78. Vafaei, A.; Ahmed, K.; Mather, P. Board Diversity and Financial Performance in the Top 500 Australian Firms. Aust. Account. Rev. 2015, 25, 413-427. [CrossRef]

79. Kennedy, P. A Guide to Econometrics; Blackwell: Oxford, UK, 1992. 
80. Amihud, Y.; Lev, B. Risk Reduction as a Managerial Motive for Conglomerate Mergers. Bell J. Econ. 1981, 12, 605. [CrossRef]

81. Farrell, K.A.; Hersch, P.L. Additions to corporate boards: The effect of gender. J. Corp. Financ. 2005, 11, 85-106. [CrossRef]

82. Chen, J.; Leung, W.S.; Song, W.; Goergen, M. Why female board representation matters: The role of female directors in reducing male CEO overconfidence. J. Empir. Financ. 2019, 53, 70-90. [CrossRef]

83. Peij, S.C.; Bezemer, P.; Maassen, G.F. The effectiveness of supervisory boards: An exploratory study of challenges in Dutch boardrooms. Int. J. Bus. Gov. Ethics 2012, 7, 191. [CrossRef]

84. Post, C.; Byron, K. Women on Boards and Firm Financial Performance: A Meta-Analysis. Acad. Manag. J. 2015, 58, 1546-1571. [CrossRef]

85. Adams, R.B.; Funk, P. Beyond the glass ceiling: Does gender matter? Manag. Sci. 2012, 58, $219-235$. [CrossRef]

86. Bianco, M.; Ciavarella, A.; Signoretti, R. Women on corporate boards in Italy: The role of family connections. Corp. Gov. Int. Rev. 2015, 23, 129-144. [CrossRef]

(C) 2020 by the authors. Licensee MDPI, Basel, Switzerland. This article is an open access article distributed under the terms and conditions of the Creative Commons Attribution (CC BY) license (http://creativecommons.org/licenses/by/4.0/). 